\title{
Phase Field Modeling of Microstructure Evolution and Concomitant Effective Conductivity Change in Solid Oxide Fuel Cell Electrodes
}

\author{
Yinkai Lei ${ }^{\mathrm{a}, *}$, Tian-Le Cheng ${ }^{\mathrm{a}, \mathrm{b}}$, You-Hai Wen ${ }^{\mathrm{a}, *}$ \\ ${ }^{a}$ National Energy Technology Laboratory, Albany, Oregon, 97321, USA \\ ${ }^{b}$ AECOM, P.O. Box 1959, Albany, OR 97321, USA
}

\begin{abstract}
Microstructure evolution plays an important role in the performance degradation of SOFC electrodes. In this work, we propose a much improved phase field model to simulate the microstructure evolution in the electrodes of solid oxide fuel cell. We demonstrate that the tunability of the interfacial energy in this model has been significantly enhanced. Parameters are set to fit for the interfacial energies of a typical Ni-YSZ anode, an LSM-YSZ cathode and an artificial reference electrode, respectively. The contact angles at various triple junctions and the microstructure evolutions in two dimensions are calibrated to verify the model. As a demonstration of the capabilities of the model, three dimensional microstructure evolutions are simulated applying the model to the three different electrodes. The time evolutions of grain size and triple phase boundary density are analyzed. In addition, a recently proposed bound charge successive approximation algorithm is employed to calculate the effective conductivity of the electrodes during microstructure evolution. The effective conductivity of all electrodes are found to decrease during the microstructure evolution, which is attributed to the increased tortuosity and the loss of percolated volume fration of the electrode phase.
\end{abstract}

Keywords: SOFC, Microstructure evolution, Phase-field simulation, Enhanced

\footnotetext{
*Corresponding author. Tel. +15419184418. Fax. +15419184493.

Email addresses: Yinkai.Lei@netl.doe.gov (Yinkai Lei ), Tianle.Cheng@netl.doe.gov (Tian-Le Cheng), Youhai.Wen@netl.doe.gov (You-Hai Wen )
}

Preprint submitted to Elsevier

January 13, 2017

(C) 2017. This manuscript version is made available under the Elsevier user license http://www.elsevier.com/open-access/userlicense/1.0/ 
interfacial energy tunability, Effective conductivity, BCSA algorithm

\section{Introduction}

Solid oxide fuel cells (SOFCs) have attracted a great deal of research attentions recently for their potential to generate electricity with high energy conversion efficiency [1-3], low pollutant emission [3, 4], and excellent fuel flexibility [5-7]. In SOFCs, the electrolyte is a conductor of oxygen ions, e.g. yttria stabilized zirconia (YSZ) or Gd doped $\mathrm{CeO}_{2}$ [8-10], while the electrode is composed of three phases, i.e. pore, electrolyte, and electrode phase. Commonly used electrode phase is both a conductor of electrons and a catalyst of either oxygen reduction reaction at cathode, e.g. $\mathrm{La}_{1-x} \mathrm{Sr}_{x} \mathrm{MnO}_{3}$ (LSM), or hydrogen oxidation reaction at anode, e.g, $\mathrm{Ni}[8]$. The oxygen molecule is reduced to the oxygen ions at the triple phase boundary (TPB) in cathode, then diffuse through the electrolyte layer and oxidize the fuel at the TPB in anode.

Due to the large activation energy of oxygen ion diffusion in the electrolyte of SOFCs $(\sim 1 \mathrm{eV})[11]$, the operating temperature of SOFC is relatively high, e.g. $600{ }^{\circ} \mathrm{C}$ to $1000{ }^{\circ} \mathrm{C}$ [1]. At such high temperatures, capillary-driven microstructure evolution, such as grain growth through grain boundary motion and particle coarsening through surface and interphase boundary motion, is significant. The thermal aging test on YSZ-Ni anodes shows that the average radius of $\mathrm{YSZ}$ and $\mathrm{Ni}$ particles increased by $\sim 200 \mathrm{~nm}$ and $\sim 300 \mathrm{~nm}$ after 500 hours at $1150{ }^{\circ} \mathrm{C}$. Projecting these data to $800{ }^{\circ} \mathrm{C}$, it has been predicted that the TPB density will decrease by $15 \% \sim 45 \%$ after 100,000 hours [12]. Ostwaldripening due to gas phase transportation or vacancy diffusion is considered to be an important mechanism of the Ni coarsening in SOFC anode $[13,14]$.

In YSZ-LSM cathodes, the prevalent grain sizes of YSZ and LSM are found to be increased from $1.18 \mu \mathrm{m}$ and $1.13 \mu \mathrm{m}$ to $1.41 \mu \mathrm{m}$ and $1.74 \mu \mathrm{m}$ respectively at $1450{ }^{\circ} \mathrm{C}$ after 100 hours [15], and the particle coarsening is found to be one of the characteristic degradation mechanisms above $850{ }^{\circ} \mathrm{C}$ among others including Mn-ion migration and formation of $\mathrm{m}-\mathrm{ZrO}_{2}$ [16]. Moreover, it has 
been reported that the particle coarsening leads to increased Ohmic resistance in both cathodes and anodes [17-20]. Since both the TPB density and Ohmic resistance are key factors to the performance of SOFC, the ability to predict the microstructure evolution in SOFC electrodes is critical to understand the long-term degradation of SOFCs.

Several phase field models have been proposed to phenomenologically simulate the time evolution of the microstructure and properties of SOFC electrodes. One of the common problems is that some models have ignored the effect of grain boundary on microstructure evolutions [21-24]. Thus, the grain growth in SOFC electrodes cannot be investigated in these models, and the grain boundary effect on the evolution of the critical properties of SOFC electrodes such as TPB density is unclear yet. Another problem is that YSZ phase has been forced to be static in some models [21, 25]. Indeed, the evolution of YSZ phase is relatively slow at operational temperatures, but the size change in YSZ phase is still observable [12, 26] and can affect the Ni coarsening in YSZ-Ni anode [26]. Therefore, a multiphase-multigrain model that treats all the interfaces in SOFC electrodes in a universal framework is needed for the simulation of microstructure evolution in SOFC electrodes. Li et al has proposed a multiphase-multigrain model that has the potential to be applied to a wide range of SOFC electrode materials [27-30]. However, the tunability of the interfacial energy in their model is limited as discussed in Appendix A. Some improvements to their model are essential to realize its full potential in simulating multigrain-multiphase systems.

In this work, we propose an improved phase field model with significantly enhanced tunability of interfacial energies. By developing three sets of parameters for anode, cathode and a reference electrode respectively, we show that this model treats all the interfaces in SOFC electrodes in a universal phase field framework, which makes it feasible to study interaction/coupling between phase boundary evolution and grain boundary evolution. The phase field model has been validated by two-dimensional (2D) simulations on the contact angles at various triple junctions and capillary-driven microstructure evolutions under 
different conditions. In addition, we generalize a recently proposed bound charge successive approximation (BCSA) algorithm [31] to evaluate the effective conductivity of the electrode, which allows monitoring the concomitant evolution of the effective conductivity directly from the real-time simulated microstructure. Since the BCSA algorithm is also based on diffuse-interface description, the two algorithms are perfectly compatible with each other, avoiding error caused by translation between sharp-interface and diffuse-interface models. The threedimensional (3D) microstructure evolution and the concomitant effective conductivity change of all three electrodes have been simulated to demonstrate the capability of the model in investigating the degradation of SOFC electrodes.

\section{Methodology}

\subsection{Phase field model}

Three major phases in SOFC electrodes, i.e. the electrolyte (YSZ), electrode (Ni/LSM) and pore phase, are considered in this work. The phase fraction of each phase is represented by a composition order parameter (COP) $C_{i}(0 \leq$ $\left.C_{i} \leq 1\right)$, where $C_{1}, C_{2}$ and $C_{3}$ denote the fraction of the electrolyte, electrode and pore phase respectively. Since $C_{1}+C_{2}+C_{3}=1$, two independent COPs $C_{1}$ and $C_{2}$ are sufficient to characterize the system. In addition to COPs, for each solid phase $i$, there are $M_{i}$ grain order parameters (GOPs) $\eta_{j}^{i}\left(0 \leq \eta_{j}^{i} \leq 1\right)$ representing grains with different crystallographic orientations. In this setup, a point located in a grain with orientation $k$ of a solid phase $i$ is represented by $C_{i}=1, \eta_{k}^{i}=1$ and $C_{j}=\eta_{l}^{i}=\eta_{l}^{j}=0$ for all $j \neq i$ or $l \neq k$. A point located in a pore is represented by $C_{i}=\eta_{k}^{j}=0$ for all $i, j$ and $k$.

The total free energy is constructed to be

$F\left(\left\{C_{i}\right\},\left\{\eta_{j}^{i}\right\}\right)=\int\left[f_{0}\left(C_{i}, \eta_{j}^{i}\right)+\sum_{i=1}^{2} \sum_{j=1}^{M_{i}} \frac{\kappa_{\eta}^{i j}}{2}\left(\nabla \eta_{j}^{i}\right)^{2}+\sum_{i=1}^{2} \sum_{j=i}^{2} \frac{\kappa_{C}^{i j}}{2}\left(\nabla C_{i} \cdot \nabla C_{j}\right)\right] d \mathbf{v}$

where $\kappa_{C}^{i j}$ and $\kappa_{\eta}^{i j}$ are the gradient energy coefficients, which need to be determined for a specific material system. $f_{0}$ is the local free energy density which 
is a function of $C_{i}$ and $\eta_{j}^{i}$, i.e.

$$
\begin{aligned}
f_{0} & =\sum_{i=1}^{2} \rho_{i} f_{1}\left(C_{i}\right)+\rho_{12} f_{1}\left(C_{1}+C_{2}\right)+\sum_{i=1}^{2} \chi_{i}\left[f_{2}\left(C_{i}\right)+\sum_{j=1}^{M_{i}} f_{3}\left(C_{i}, \eta_{j}^{i}\right)\right] \\
& +\sum_{i=1}^{2} \sum_{j=1}^{M_{i}} \sum_{k>j}^{M_{i}} \epsilon_{j k}^{i} f_{4}\left(\eta_{j}^{i}, \eta_{k}^{i}\right)
\end{aligned}
$$

where

$$
\begin{aligned}
& f_{1}(C)=C^{2}(1-C)^{2} \\
& f_{2}(C)=\frac{1}{4} C^{4} \\
& f_{3}(C, \eta)=-\frac{1}{2} C^{2}\left(2 \eta^{3}-\eta^{4}\right)+\frac{1}{4} \eta^{4} \\
& f_{4}\left(\eta_{j}, \eta_{k}\right)=\frac{1}{2}\left(\eta_{j}\right)^{2}\left(\eta_{k}\right)^{2}
\end{aligned}
$$

Here $f_{1}$ is a function of COP that accounts for the coupling among the three phases, i.e. the electrode, electrolyte and pore phase, $f_{2}(C)$ and $f_{3}(C, \eta)$ describe the coupling between a COP and the GOPs of the corresponding phase, and $f_{4}\left(\eta_{i}, \eta_{j}\right)$ the interaction between different GOPs in the same phase. In Eqs. $3, f_{1}\left(C_{1}\right), f_{1}\left(C_{2}\right)$ and $f_{1}\left(C_{1}+C_{2}\right)$ keep the pair $\left(C_{1}, C_{2}\right)$ to be around $(1,0),(0,1)$ or $(0,0)$, which corresponds to the electrolyte, electrode and pore phase respectively. $f_{4}\left(\eta_{i}, \eta_{j}\right)$ is used to account for the grain boundary energy between two grains. This grain boundary energy needs to be vanished in pore phase, in which grain orientation becomes meaningless. Therefore, when $C_{i}=0$ (pore phase), it is necessary to have $\eta_{j}^{i}=0$ to make $f_{4}\left(\eta_{i}, \eta_{j}\right)$ vanish from the bulk free energy density. When $C_{i}=1$, however, $\eta_{j}^{i}$ must have a positive value, so that $f_{4}\left(\eta_{i}, \eta_{j}\right)$ is not vanished at grain boundaries. Therefore, the combination of $f_{2}(C)$ and $f_{3}(C, \eta)$ is used to keep $\eta_{j}^{i}=0$ and 1 when $C_{i}=0$ and 1 respectively. We plot the projection of $f_{0}$ on $C_{1}-\eta_{1}^{1}$ plane when $C_{2}=\eta_{i}^{2}=0$ in Fig. 1(a). It shows that $f_{0}$ only has two minima at $C_{1}=\eta_{1}^{1}=1$ and $C_{1}=\eta_{1}^{1}=0$ respectively on this plane, which demonstrates the effect of $f_{2}(C)$ and $f_{3}(C, \eta)$. The projection of $f_{0}$ on the $C_{1}-C_{2}$ plane when $C_{1}=\eta_{1}^{1}$ and $C_{2}=\eta_{1}^{2}$, and the $\eta_{1}^{1}-\eta_{2}^{1}$ plane when $C_{1}=1$ and $C_{2}=\eta_{i}^{2}=0$ are also given in 


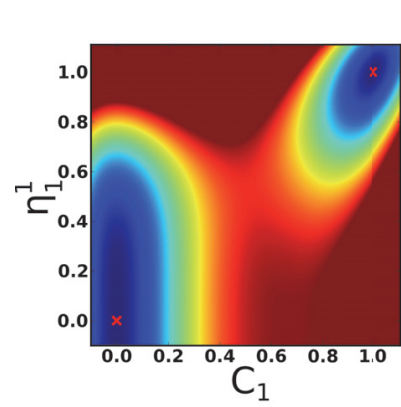

(a)

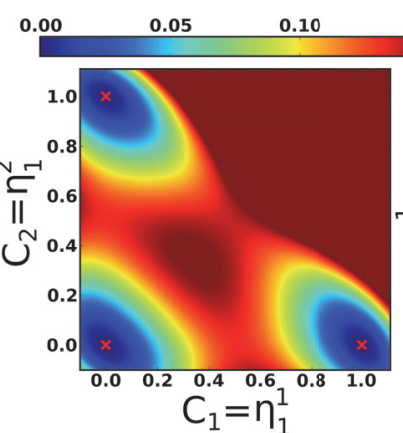

(b)

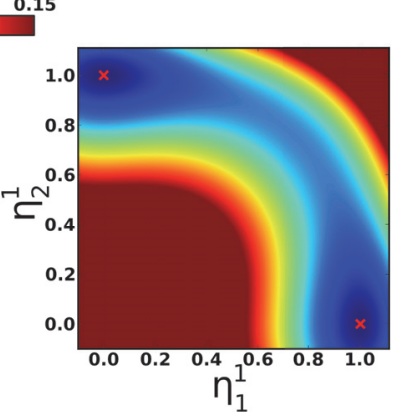

(c)

Figure 1: Free energy density $f_{0}$ projected on (a) $C_{1}-\eta_{1}^{1}$ plane when $C_{2}=\eta_{i}^{2}=0$, (b) $C_{1}-C_{2}$ plane when $C_{1}=\eta_{1}^{1}$ and $C_{2}=\eta_{1}^{2}$ and (c) $\eta_{1}^{1}-\eta_{2}^{1}$ plane when $C_{1}=1$ and $C_{2}=\eta_{i}^{2}=0$. The red crosses mark the local minima which correspond to the existing phases. (For color version of this figure, the reader is referred to the online version of this article.)

Figs. 1(b) and 1(c). It shows that $f_{0}$ has local minima only at $C_{i}=\eta_{j}^{i}=0$ or $C_{i}=1, \eta_{j}^{i}=1, C_{k}=\eta_{l}^{i}=\eta_{m}^{k}=0, k \neq i, l \neq j$. Again, the phenomenological parameters $\rho_{i}, \rho_{12}, \chi_{i}$ and $\epsilon_{j k}^{i}$ need to be determined for a specific material system under investigation.

Compared to the model in Refs. $[27,28,32]$, the term $\frac{\kappa_{C}^{12}}{2}\left(\nabla C_{1} \cdot \nabla C_{2}\right)$ and $\rho_{12} f_{1}\left(\sum_{i=1}^{2} C_{i}\right)$ have been added to the free energy density functional, while the formula of $f_{3}(C, \eta)$ has been modified. The purpose of these modifications is to overcome the tunability problem in the old model. As shown in Appendix A, the energy of the interphase boundary in the old model is always close to the summation of the surface energy of the two phases forming the interphase boundary. Those two added terms are essential to make the energy of the interphase boundary tunable. Similar terms have been used in previous phase field simulations of multi-phase system $[22,23,33,34]$. In addition, the GOP $\eta$ in the old model are allowed to be either +1 or -1 in the solid phases, which may cause unphysical high-energy grain boundary when two grains with $\eta=1$ and $\eta=-1$ are in contact with each other (see the discussion in Appendix A). The cubic term in $f_{3}(C, \eta)$ removes the local minimum at $C=1$ and $\eta=-1$, thus eliminates this unphysical high-energy grain boundary.

The driving force for microstructure evolution is derived by taking the vari- 
ation of the total free energy with respect to COPs and GOPs. There are experimental evidences showing that the total phase fraction of each phase is nearly conserved during particle coarsening [12, 35], while the grain orientations are not conserved to allow grain rotation. The spatial-temporal evolution of the COP $C_{i}$ and GOP $\eta_{j}^{i}$ are governed by the time-dependent Cahn-Hilliard diffusion equation and Ginzburg-Landau equation respectively, i.e.

$$
\begin{aligned}
\frac{\partial C_{i}}{\partial t} & =\nabla \cdot\left\{M_{C}^{i} \nabla\left[\frac{\partial f_{0}}{\partial C_{i}}-\sum_{i=1}^{2} \kappa_{C}^{i j} \nabla^{2} C_{i}\right]\right\} \quad i=1,2 \\
\frac{\partial \eta_{j}^{i}}{\partial t} & =-M_{\eta}^{i j}\left(\frac{\partial f_{0}}{\partial \eta_{j}^{i}}-\kappa_{\eta}^{i j} \nabla^{2} \eta_{j}^{i}\right) \quad i=1,2 \text { and } j=1,2, \ldots, M_{i}
\end{aligned}
$$

where $M_{C}^{i}$ and $M_{\eta}^{i j}$ are the mobility of the COP and GOP respectively. In general, $M_{C}^{i}$ and $M_{\eta}^{i j}$ are second order tensors. But the grain growth and particle coarsening is assumed to be isotropic in this work, in which case $M_{C}^{i}$ and $M_{\eta}^{i j}$ are just scalars.

In the current phase field model, the COP $C_{i}$ and GOP $\eta_{j}^{i}$ are dimensionless, while the unit of all the energetic parameters, i.e. $\rho_{i}, \rho_{12}, \chi_{i}, \epsilon_{j k}^{i}, \kappa_{C}^{i j}$ and $\kappa_{\eta}^{i j}$, and kinetic parameters, i.e. $M_{C}^{i}$ and $M_{\eta}^{i j}$, can be derived from the unit of length, time and energy density. Here, we set the unit of length, time and energy density to be $d_{0}, t_{0}$ and $e_{0}$, respectively. The corresponding unit of all the parameters are given in Table 1. In this unit scheme, the dimensionless form of the kinetic equations of $C_{i}$ and $\eta_{j}^{i}$ are the same as those in Eqs. 4 and 5. Once $M_{C}^{i}$ and $M_{\eta}^{i j}$ are known, the real time span corresponding to a certain simulation time can be derived from Table 1 for a given grain size of the initial microstructure.

Table 1: Units of all the parameters in current phase field model.

\begin{tabular}{ccccccc}
\hline length & time & energy density & $\rho_{i}, \rho_{12}, \chi_{i}, \epsilon_{j k}^{i}$ & $\kappa_{C}^{i j}, \kappa_{\eta}^{i j}$ & $M_{C}^{i}$ & $M_{\eta}^{i j}$ \\
\hline$d_{0}$ & $t_{0}$ & $e_{0}$ & $e_{0}$ & $e_{0} d_{0}^{2}$ & $d_{0}^{2} t_{0}^{-1} e_{0}^{-1}$ & $t_{0}^{-1} e_{0}^{-1}$ \\
\hline
\end{tabular}

Finite difference scheme is used to solve Eqs. 4 and 5. The spatial part is discretized on a cubic mesh with $d x=d y=d z=d_{0}$, while the time integration is carried out by the fourth order Runge-Kutta method with $d t=0.01 t_{0}$. 
The modeling of polycrystals requires sufficient number of GOP $\eta_{j}^{i}$ to be used in each phase, which leads to a huge number of coupled equations in Eq. 5. This difficulty is overcome by adopting the sparse data structure proposed in Refs. [36] and [37]. DREAM.3D [38] is used to generate initial microstructures with equiaxed grains. Ten initial microstructures are generated for each of three compositions, i.e. 35\% YSZ-35\% Ni/LSM-30\%pore, 30\%YSZ-40\% Ni/LSM$30 \%$ pore and $40 \%$ YSZ-30\%Ni/LSM-30\%pore, for the purpose of exploring the effect of the initial structure on the time evolution of microstructure and effective conductivity. These three compositions are chosen so that all three phases can form percolated network in the simulation cell.

In the current phase field model, the interfacial energy reduction is the driving force for the microstructure evolution. Therefore, it is critical to link the interfacial energy of the model to that of the real materials. There are five types of interface in the electrolyte-electrode-pore composite system, namely, the surface of the electrolyte and electrode phase, the grain boundary (GB) of the electrolyte and electrode phase, and the interphase boundary (IPB) between the electrolyte and electrode phase. As shown in Appendix A, the energy and the width of a flat interface depend on both the gradient coefficient parameters $\kappa_{C}^{i j}$ and $\kappa_{\eta}^{i j}$ and the parameters $\rho_{i}, \rho_{12}, \chi_{i}$ and $\epsilon_{j k}^{i}$ in the local free energy density functional described in Eq. 2. So we develop two sets of parameters to reproduce the relative interfacial energy of YSZ-LSM (for cathode) and YSZ-Ni (for anode) material systems. In addition, another set of parameters is developed for a reference material system in which the energies of all five interfaces are the same. The detailed discussion of the interfacial energies in these three material systems is given in Sec. 3.1.

The mobility $M_{C}^{i}$ and $M_{\eta}^{i j}$ are the key parameters to link the results of phase field simulation to experimental results. The mobility of $\operatorname{COP} M_{C}^{i}$ is related to the atomic diffusivity $D_{i}$ by [39]

$$
M_{C}^{i}=\frac{D_{i} V_{m}}{R T}
$$

where $V_{m}$ and $T$ are the molar volume $\left(\sim 10^{-6} \mathrm{~m}^{3}\right.$ for solid phase $)$ and temper- 
ature respectively. The mobility of GOP $M_{\eta}^{i j}$ is related to the rate constant $k$ for grain growth (assuming ideal grain growth) by [37]

$$
M_{\eta}^{i j} \sim \frac{k}{\delta_{G B} \gamma_{G B}}
$$

where $\delta_{G B}$ is the width of GB in the phase field simulation, which is usually several $d_{0}$, and $\gamma_{G B}$ is the GB energy, which is $\sim 1 \mathrm{~J} \mathrm{~m}^{-2}$ for all three phases [40-43]. At $1000{ }^{\circ} \mathrm{C}$, the self-diffusivity of $\mathrm{Ni}\left(\sim 10^{-16} \mathrm{~m}^{2} \mathrm{~s}^{-1}\right)$ [44] is comparable to the cation diffusivity in $\operatorname{LSM}\left(10^{-15} \sim 10^{-14} \mathrm{~m}^{2} \mathrm{~s}^{-1}\right)$ [45], both are several orders of magnitude greater than the cation diffusivity in YSZ $\left(10^{-24} \sim 10^{-23} \mathrm{~m}^{2} \mathrm{~s}^{-1}\right)[46]$. The rate constant for grain growth in YSZ is unknown yet. The rate constant for grain growth in $\operatorname{LSM}\left(\sim 10^{-18} \mathrm{~m}^{2} \mathrm{~s}^{-1}\right)[47]$ is several orders of magnitude smaller than that in pure $\mathrm{Ni}\left(\sim 10^{-12} \mathrm{~m}^{2} \mathrm{~s}^{-1}\right)$ [48]. However, this large rate constant for grain growth has only been found in highly pure $\mathrm{Ni}(99.999 \%)$, and it is largely reduced in less pure $\mathrm{Ni}$, e.g. $99.5 \% \mathrm{Ni}$ [48]. It is known that a tiny amount of impurities can lower the GB mobility of metals by several orders of magnitude due to solute drag effect [49]. Therefore, we anticipate that the rate constant for grain growth in $\mathrm{Ni}$ in $\mathrm{SOFC}$ electrode is several orders of magnitude lower than $10^{-12} \mathrm{~m}^{2} \mathrm{~s}^{-1}$. For simplicity, we assume that the mobility of GOPs of Ni is equal to that of LSM. We set the mobility of COP $M_{C}^{i}$ to be 0.6 and the mobility of GOP $M_{\eta}^{i j}$ to be 2.5 for all phases and grain orientations. When $d_{0}=0.1 \mu \mathrm{m}, t_{0}=1000 \mathrm{~s}$ and $e_{0}=10^{8} \mathrm{~J} \mathrm{~m}^{-3}$, this corresponds to an atomic diffusivity of $\sim 10^{-15} \mathrm{~m}^{2} \mathrm{~s}^{-1}$, which is comparable to the self-diffusivity of $\mathrm{Ni}$ and cation diffusivity in LSM, and a rate constant for ideal grain growth of $\sim 10^{-17} \mathrm{~m}^{2} \mathrm{~s}^{-1}$, which is comparable to the rate constant of ideal grain growth in LSM. The simplified mobility setting makes the coarsening rate of YSZ overestimated, and such effect is discussed in Sec. 5.1.

\subsection{Effective Conductivity}

The effective conductivity of the electrode is calculated by solving the Poisson's equation

$$
\nabla \cdot(\sigma(\mathbf{r}) \nabla \phi(\mathbf{r}))=0
$$


where $\sigma(\mathbf{r})$ and $\phi(\mathbf{r})$ are the spatial distribution of the local conductivity and electric potential, respectively, in the electrode. Imposing an electric potential difference $U$ between the upper and lower boundary of the electrode in $z$-direction, the effective conductivity can be calculated by

$$
\sigma_{e f f}=\frac{I}{U} \frac{L}{A}
$$

where $L$ and $A$ are the length and cross-section area of the electrode and $I$ is calculated by

$$
I=\int_{\partial \Omega_{U}} \sigma(\mathbf{r}) \mathbf{n} \cdot \nabla \phi(\mathbf{r}) d A=\int_{\partial \Omega_{L}} \sigma(\mathbf{r}) \mathbf{n} \cdot \nabla \phi(\mathbf{r}) d A
$$

where $\mathbf{n}$ is the normal vector of the upper $\left(\partial \Omega_{U}\right)$ and lower $\left(\partial \Omega_{L}\right)$ boundary of the electrode in $z$-direction.

In this work, we generalize the BCSA algorithm to solve Eq. 8. The original BCSA algorithm was developed to solve the Poisson's equation in the electrostatic and magnetostatic problems [31]. The idea is to decompose Eq. 8 with spatial varying $\sigma(\mathbf{r})$ into two equations, i.e.

$$
\sigma_{0} \nabla^{2} \phi(\mathbf{r})=g(\mathbf{r})
$$

and

$$
\nabla \cdot\left(\sigma^{\prime}(\mathbf{r}) \nabla \phi(\mathbf{r})\right)=-g(\mathbf{r})
$$

where $\sigma_{0}$ is a constant which could affect the accuracy and convergence of the algorithm, $\sigma^{\prime}(\mathbf{r})$ is the difference between $\sigma(\mathbf{r})$ and $\sigma_{0}$. In the original BCSA algorithm, the term mathematically equivalent to $-g(\mathbf{r})$ has a physical meaning of local bound charge density. Here, it is difficult to tell the clear physical meaning of $g(\mathbf{r})$, which is but unimportant. The mathematical equivalence allows the current algorithm converges at the same rate as the original BCSA for electrostatics/magnetostatics. Eqs. 11 and 12 are solved iteratively: starting from an initial guess of $g(\mathbf{r}), \phi(\mathbf{r})$ is first obtained by solving Eq. 11; then, from this $\phi(\mathbf{r})$, Eq. 12 is used to calculate a new $g^{\prime}(\mathbf{r})$, which is mixed with $g(\mathbf{r})$ for the current iteration to generate the $g(\mathbf{r})$ for the next iteration, i.e.

$$
g^{N+1}(\mathbf{r})=g^{N}(\mathbf{r})+\lambda\left[g^{\prime}(\mathbf{r})-g^{N}(\mathbf{r})\right]
$$


The iteration is stopped when the relative change in $g(\mathbf{r})$ is less than a predefined number $\delta_{g}$ which controls the target precision. The choice of $\lambda$ also affects the convergence of the algorithm. $\lambda$ is generally related to the contrast of local conductivity in the domain [31]. More detailed discussion on the choice of $\sigma_{0}$ and $\lambda$ would be published elsewhere. In this work, we choose $\sigma_{0}=1$ and $\lambda=0.5 / \sigma_{\max }$, where $\sigma_{\max }$ is the maximum value of $\sigma^{\prime}(\mathbf{r})$. Gauss-Seidel iterative method is used to solve Eq. 11. If periodic boundary condition is appropriate, fast Fourier transform can be invoked to solve Eq. 11 more efficiently [31].

The spatial varying local conductivity $\sigma(\mathbf{r})$ is obtained by interpolating the bulk conductivity of the electrolyte and electrode phase on the simulated microstructures.

$$
\sigma(\mathbf{r})= \begin{cases}\sigma_{Y S Z}, & C_{1}(\mathbf{r})>C_{2}(\mathbf{r}) \& C_{1}(\mathbf{r})>R \\ \sigma_{N i / L S M}, & C_{1}(\mathbf{r})<C_{2}(\mathbf{r}) \& C_{2}(\mathbf{r})>R \\ 0, & C_{1}(\mathbf{r})<R \& C_{2}(\mathbf{r})<R\end{cases}
$$

where $\sigma_{Y S Z}$ and $\sigma_{N i / L S M}$ are the bulk conductivity of YSZ and Ni/LSM respectively, and $R$ is a cutoff of COP. It is found that $R$ affects the value of effective conductivity but has little effect on its time evolution. Therefore, we simply set $R$ to be 0.4 . GB conductivity of YSZ is greater than bulk conductivity $[50,51]$. In this work, the GB conductivity is virtually averaged out over the bulk conductivity by using the conductivity of polycrystalline samples for all phases, i.e. $11.54 \mathrm{~S} \mathrm{~m}^{-1}$ [50] for YSZ, $2.4 \times 10^{6} \mathrm{~S} \mathrm{~m}^{-1}$ for $\mathrm{Ni}$ [52] and $8000 \mathrm{~S} \mathrm{~m}^{-1}$ for LSM [53]. Such homogenization procedure could lead to missing of grain size effect on the effective conductivity during grain growth. But for YSZ the grain size effect is only significant for sub-micron size grains [51]. Since in this work we mainly model the late-stage coarsening and the starting grain size is already on the micron level, the size effect is ignored. However, the combination of phase-field modeling and BCSA algorithm used in this work is still applicable to sub-micron and nano-crystalline YSZ, in which case an explicit term accounted for the GB conductivity should be added to Eq. 14 .

The huge conductivity contrast between Ni and YSZ leads to a slow conver- 
gence of BCSA algorithm in anode [31]. However, all phases form percolated network at the chosen compositions in our simulations. The effective conductivity of the anode is dominated by the electronic conductivity of $\mathrm{Ni}$ to the large conductivity difference between YSZ and Ni. We find that the ratio between the effective conductivity and the bulk conductivity of $\mathrm{Ni}\left(\sigma_{N i}\right)$ is a constant once $\sigma_{N i}$ is sufficiently greater than the bulk conductivity of YSZ. A test simulation shows that the ratio between the effective conductivity and $\sigma_{N i}$ is 0.137 and 0.136 when $\sigma_{N i}$ is set to $10^{4} \mathrm{~S} \mathrm{~m}^{-1}$ and $10^{5} \mathrm{~S} \mathrm{~m}^{-1}$ respectively. Therefore, in the effective conductivity calculations in anode, we set $\sigma_{N i}$ to a relative smaller value, i.e. $10^{4} \mathrm{~S} \mathrm{~m}^{-1}$, to speed up the convergence of the effective conductivity calculation, then scale the result to $2.4 \times 10^{6} \mathrm{~S} \mathrm{~m}^{-1}$ to obtain the effective conductivity.

\section{Model Validations}

\subsection{Interfacial energy calibration}

The energies of all five types of flat interfaces are calibrated on a 1D model with 128 grid points. 10000 time steps are used to equilibrate the profile of the order parameters over the interface. The interfacial energy is calculated by integrating the free energy difference between the equilibrated interface and the bulk over the simulation cell. The three developed parameter sets are given in Table 2 and the corresponding relative interfacial energies are shown in Table 3. The parameters are tuned so that there are 4 points on each interface where the order parameters are between 0.02 and 0.98. It shows that the developed parameters provide a good description of the anode (YSZ-Ni) materials system, in which the energy of Ni surface and Ni-YSZ IPB is the dominating driving force for the microstructure evolution. For the cathode, due to the lack of LSMrelated interfacial energy, we simply tune the energy of LSM grain boundary to be the greatest so that it is the major driving force for the microstructure evolution as observed in experiments [15].

Furthermore, the contact angles at TPB and Ni/LSM-Ni/LSM-pore triple junction are simulated to verify the calculated relative interfacial energies as 
Table 2: The parameters for the anode (YSZ$\mathrm{Ni}$, cathode (YSZ-LSM) and reference electrode.

\begin{tabular}{cccc}
\hline & Anode & Cathode & Reference \\
\hline$\kappa_{C}^{11}$ & 0.6 & 0.6 & 0.6 \\
$\kappa_{C}^{22}$ & 1.1 & 0.5 & 0.6 \\
$\kappa_{C}^{12}$ & 0.8 & 0.6 & 0.7 \\
$\kappa_{\eta}^{1 j}$ & 0.2 & 0.2 & 0.4 \\
$\kappa_{\eta}^{2 j}$ & 0.3 & 0.7 & 0.4 \\
$\rho_{1}$ & 0.3 & 0.2 & 0.3 \\
$\rho_{2}$ & 1.0 & 0.3 & 0.3 \\
$\rho_{12}$ & 0.7 & 0.7 & 0.7 \\
$\chi_{1}$ & 0.4 & 0.4 & 0.4 \\
$\chi_{2}$ & 0.4 & 0.4 & 0.4 \\
$\epsilon_{j k}^{1}$ & 0.5 & 0.5 & 1.2 \\
$\epsilon_{j k}^{2}$ & 0.6 & 1.2 & 1.2 \\
\hline
\end{tabular}

Table 3: The simulated relative interfacial energies with respect to the surface energy of YSZ $\gamma_{s}^{Y S Z}$ for the anode (YSZ-Ni), cathode (YSZ-LSM) and reference electrode. The available experimental values are given in bracket.

\begin{tabular}{ccccc}
\hline$\gamma / \gamma_{s}^{Y S Z}$ & YSZ GB & Ni/LSM surface & Ni/LSM GB & YSZ-Ni/LSM IPB \\
\hline Anode & $0.6\left(0.5^{\mathrm{a}}\right)$ & $1.6\left(1.7^{\mathrm{b}}\right)$ & $0.8\left(0.8^{\mathrm{c}}\right)$ & $1.5\left(1.5 \sim 1.7^{\mathrm{d}}\right)$ \\
Cathode & $0.6\left(0.5^{\mathrm{a}}\right)$ & 1.1 & 1.5 & 1.1 \\
Reference & 1.0 & 1.0 & 1.0 & 1.0 \\
\hline
\end{tabular}

${ }^{\text {a }}$ Ref. [40]

b Ref. [41]

${ }^{c}$ Ref. [42]

${ }^{\mathrm{d}}$ Ref. [43]

shown in Fig. 2. The TPB has been calibrated in most previous phase field simulations $[21,23,27]$ but the Ni/LSM-Ni/LSM-pore triple junction has only been calibrated in Ref. [25] before. In Fig. 2(a) of TPB in the anode, it shows that the contact angles opposite to YSZ surface, Ni surface and YSZ-Ni IPB are $138^{\circ}, 105^{\circ}$ and $117^{\circ}$ respectively, which indicates that $\mathrm{Ni}$ surface has the greatest energy. In Figs. 2(b) and 2(c), it shows that the three contact angles at TPB are all around $120^{\circ}$ in the cathode and reference electrode, implying that the energy of YSZ surface, LSM surface and YSZ-LSM IPB are similar. At the Ni/LSM-Ni/LSM-pore triple junctions shown in Figs. 2(d)-(f), the contact angles between two surfaces of electrode phase are $148^{\circ}, 100^{\circ}$ and $120^{\circ}$ in the 
anode, cathode and reference electrode respectively. This reflects the significant difference in the GB energy of the electrode phase in these three electrodes. All simulated contact angles are consistent with the relative interfacial energies in Table 3, showing that the desired interfacial energetics has been incorporated into the phase field simulations.

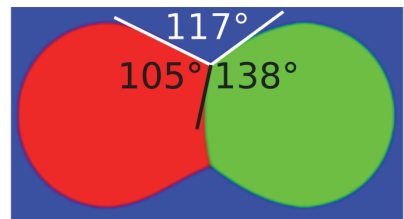

(a)

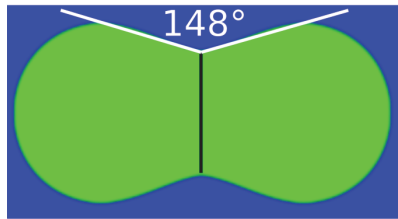

(d)

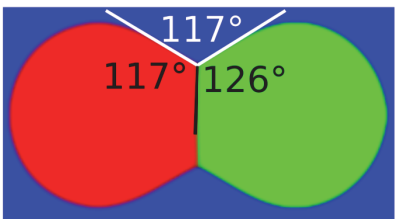

(b)

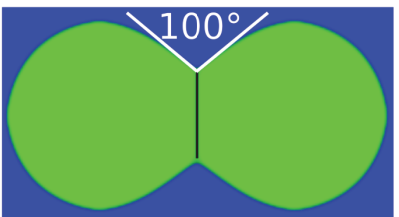

(e)

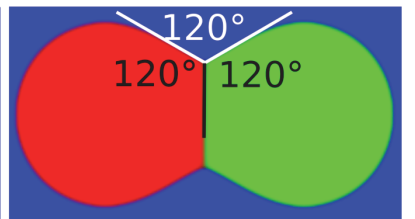

(c)

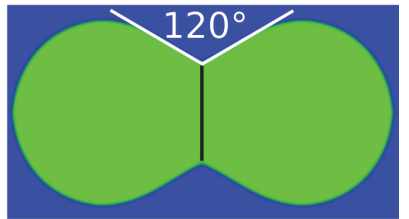

(f)

Figure 2: The contact angles at (a) TPB in the anode, (b) TPB in the cathode, (c) TPB in the reference electrode, (d) Ni-Ni-pore triple junction in the anode, (e) LSM-LSM-pore triple junction in the cathode and (f) LSM-LSM-pore triple junction in the reference electrode. The red, green and blue phase are the electrolyte (YSZ), electrode (Ni/LSM) and pore respectively. (For color version of this figure, the reader is referred to the online version of this article.)

\subsection{Capillary-driven microstructure evolution}

It is well-known that the grain size evolution of a material follows the power law

$$
\bar{R}^{n}-\bar{R}_{0}^{n}=k t
$$

where $\bar{R}$ and $\bar{R}_{0}$ are the average grain radius at time $t$ and $0, k$ is the rate constant and $n$ is the growth exponent which reflects the rate-controlling process. Previous phase field simulations show that $n$ is equal to 2 for grain growth [54] and 3 for particle coarsening due to Ostwald ripening [55, 56]. Here we calibrate the grain growth and particle coarsening of YSZ in our phase field model using the reference parameter set. A 2D model with $100 \%$ YSZ has been built to simulate the grain growth, while another 2D model with 20\% YSZ and $80 \%$ LSM has been built to simulate the particle coarsening. The initial grain size of 
YSZ is set to $0.5 \mu \mathrm{m}$. In the $20 \% \mathrm{YSZ}-80 \% \mathrm{LSM}$ model, only one GOP for LSM is used for the purpose of eliminating the effect of LSM GB. The microstructure of these two models with 250 YSZ grains are given in Figs. 3(a) and 3(b) respectively. Both simulations are stopped when the number of YSZ grains reduces to 100 .

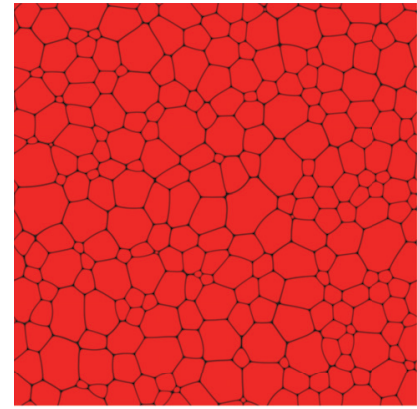

(a)

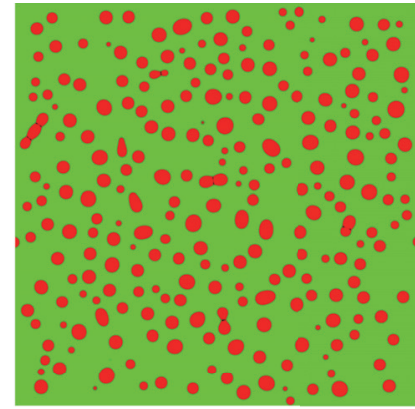

(b)

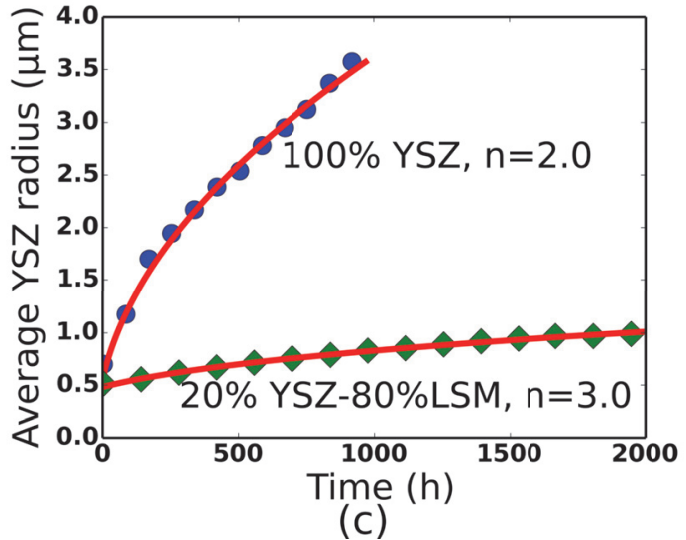

Figure 3: The 2D microstructure of the reference electrode with (a) $100 \% \mathrm{YSZ}$ and (b) $20 \%$ YSZ-80\%LSM when there are 250 YSZ grains remaining. The red and green phase are electrolyte and electrode phase respectively. (c) The grain size evolution of YSZ in 2D reference electrode with 100\% YSZ and 20\% YSZ-80\%LSM. (For color version of this figure, the reader is referred to the online version of this article.)

The grain size evolutions in these two models are given in Fig. 3(c). It shows that the grain growth is much faster than the particle coarsening, and the exponents for these two types of microstructure evolutions are around 2 and 3 respectively, which is consistent with previous phase field simulations [54-56]. The different exponent is a result of different effective diffusion length. In the 
grain growth, the order parameters only need to diffuse through the width of the GB, while in the particle coarsening, the order parameters have to diffuse through the space between grains, which, as shown in Figs. 3(a) and 3(b), is much greater than the width of GB.

\subsection{Validation of the effective conductivity calculation method}

The original BCSA for electrostatics has been validated in detail [31]. In principle, that validation should also apply to steady-state electric conduction problem due to the mathematical equivalence. For confirmation, a onedimensional conduction problem is simulated, as shown in Figs. 4(a) and 4(c), where two layered structures of YSZ-LSM are set up. The potential difference of $1 \mathrm{~V}$ has been imposed at the two ends of the structure in $z$-direction. The distribution of the electric potential and COP of YSZ and LSM in $z$-direction are given in Figs. 4(b) and 4(d). It shows that the potential difference is distributed mostly on YSZ due to its small conductivity compared to that of LSM. The calculated effective conductivity of these two structures are $23.2 \mathrm{~S} \mathrm{~m}^{-1}$ and $23.4 \mathrm{~S} \mathrm{~m}^{-1}$, both agree well with the analytical value of $23.1 \mathrm{~S} \mathrm{~m}^{-1}$.

It is known that the simulation cell must be sufficiently large to obtain reliable estimation of the effective conductivity [57, 58]. We investigate the effect of the cell size on the effective conductivity with a constant grain radius of $0.5 \mu \mathrm{m}$. The standard deviation of the average effective conductivity over 10 different microstructure is found to be decreasing with the size of the simulation cell. It is found that the standard deviation reduces to less than $5 \%$ when the size of simulation cell is $12.8 \times 12.8 \times 12.8 \mu \mathrm{m}^{3}$. This size of simulation cell is therefore used in all the simulations of the time evolution of the microstructure and effective conductivity in this work.

\section{Results}

\subsection{Grain size evolution}

Fig. 5 shows the evolution of a random initial microstructure after 40000 steps for the anode, cathode and reference electrode respectively. In real unit, 


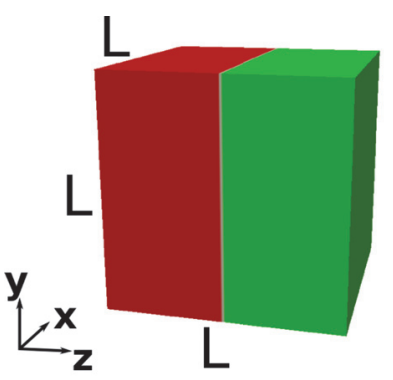

(a)

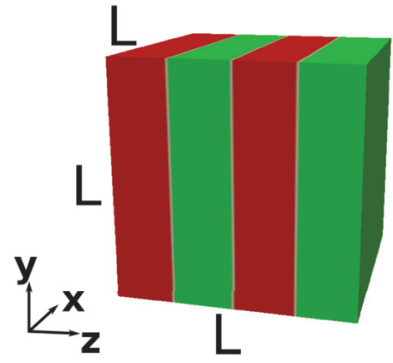

(c)

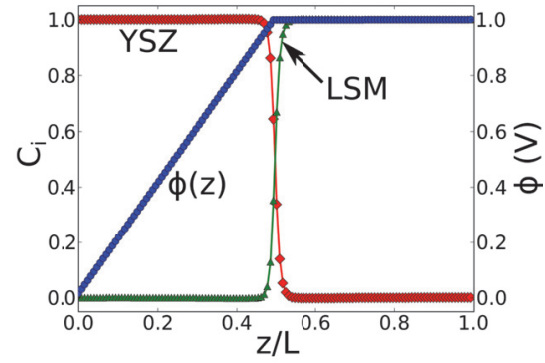

(b)

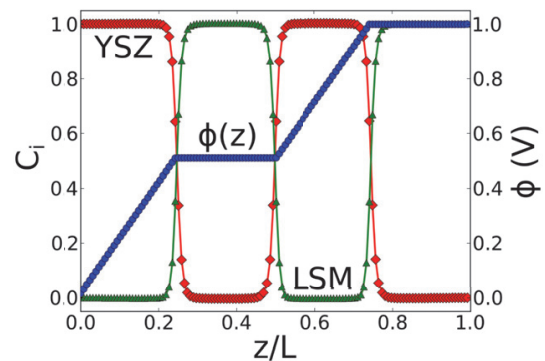

(d)

Figure 4: (a) and (c) Two layered structures of YSZ-LSM composites, the red and green phases are YSZ and LSM respectively. (b) and (d) The corresponding distribution of YSZ, LSM and electric potential in $z$-direction.(For color version of this figure, the reader is referred to the online version of this article.)

this corresponds to thermal coarsening at $1000{ }^{\circ} \mathrm{C}$ after around 110 hours. The increase in the grain size is obvious. The insets in Fig. 5 show the local evolution of YSZ and Ni/LSM GBs. It clearly shows that the YSZ GB density in the anode and cathode (Figs.5(b) and 5(c)) is greater than that in the reference electrode (Fig.5(d)). It also shows that the Ni GB density in anode is significantly higher than the LSM GB density in the cathode and reference electrode. It is found that, regardless of the composition, the average density of LSM GB in the cathode and reference electrode is about $50 \%$ and $70 \%$ lower than the density of Ni GB in the anode respectively. In addition, the density of YSZ GB in the reference electrode is found to be about $40 \%$ lower than that in the anode and cathode. This difference in GB density can be attributed to the relative interfacial energy differences given in Table 3.

The time evolution of the average grain radius of each phase in the anode and cathode with $35 \%$ YSZ, 35\% Ni/LSM and 30\% pores are given in Figs. 6(a) 


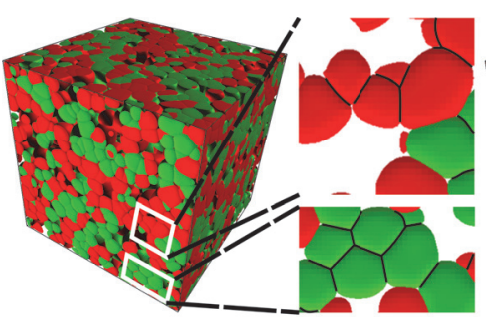

(a)

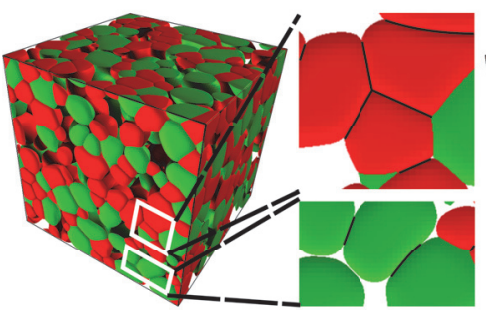

(c)

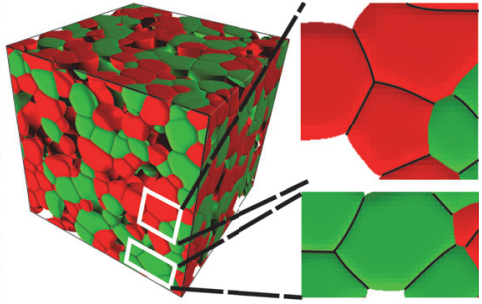

(b)

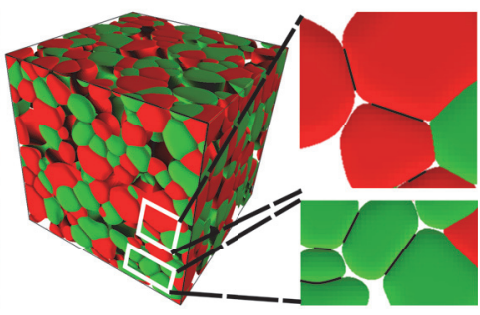

(d)

Figure 5: (a) Initial microstructure and the corresponding microstructure after 110 hours in the (b) anode, (c) cathode and (d) reference electrode. The upper and lower insets show the local evolution of YSZ and Ni/LSM grain boundary respectively. The red and green grains are electrolyte (YSZ) and electrode (Ni/LSM) respectively. The volume fraction of each phase is $35 \%$ for YSZ, $35 \%$ for Ni/LSM and 30\% for pores. (For color version of this figure, the reader is referred to the online version of this article.)

and 6(b). The evolution of the grain size in the electrodes with other compositions are similar. GOPs are used to evaluate the volume of the grain in the solid phase, and the grain radius is calculated by assuming a spherical grain. Inscribed sphere method is used to determine the radius of the pores in which the GOP is absent [59]. It shows that after 110 hours, the average grain size of $\mathrm{Ni}$ in anode and LSM in cathode increases from $\sim 0.50 \mu \mathrm{m}$ to $\sim 0.75 \mu \mathrm{m}$ and $\sim 0.80 \mu \mathrm{m}$ respectively, whereas the average grain size of YSZ in both anode and cathode increases from $\sim 0.50 \mu \mathrm{m}$ to $\sim 0.70 \mu \mathrm{m}$. It is clear that, despite the mobility of all phases are set to be the same, the coarsening of YSZ grains is slower than that of $\mathrm{Ni}$ and LSM due to its lower surface and GB energy. Electron backscatter diffraction was used in Ref. [15] to reconstruct the 3D grain-orientation distribution in LSM-YSZ cathode. The measured coarsening rate of LSM grains ( $~ 54 \%$ after 100 hours) is comparable to the prediction of our simulations ( $\sim 60 \%$ after 110 hours). But measured coarsening rate of YSZ 

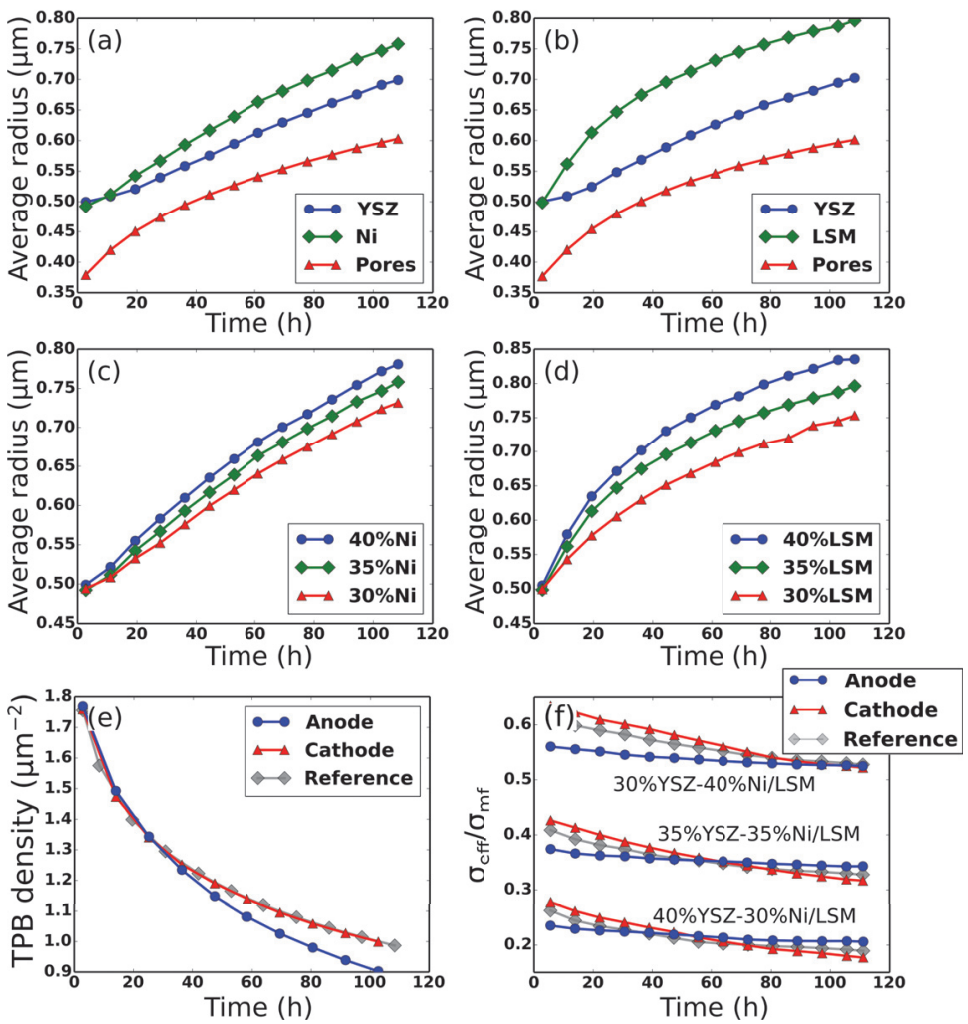

Figure 6: The grain size evolution of each phase in the (a) anode and (b) cathode with $35 \%$ YSZ, $35 \% \mathrm{Ni} / \mathrm{LSM}$ and $30 \%$ pores, and the grain size evolution of (c) $\mathrm{Ni}$ in the anode and (d) LSM in the cathode with three different compositions, (e) the time evolution of TPB density for anode, cathode and reference system with $35 \%$ YSZ, 35\% Ni/LSM and 30\% Pore, and (f) the time evolution of effective conductivity for anode, cathode and reference system at three different compositions.

grains ( $19 \%$ after 100 hours) is slower than the prediction of our simulations ( $\sim 40 \%$ after 110 hours) due to the exaggerated mobility of YSZ phase in our simulations. The simulated growth of the Ni grains is comparable to what has been found in Ref. [60], in which the grain size of $\mathrm{Ni}$ in an anode with $37 \%$ $\mathrm{Ni}$ and $40 \%$ YSZ increases from $\sim 0.50 \mu \mathrm{m}$ to $\sim 0.80 \mu \mathrm{m}$ after 92 hours at 1050 ${ }^{\circ} \mathrm{C}$. However, the Ni grain size from the experiment is determined from the reconstructed phase distribution instead of direct measurements, so it is tricky to compare these two results. Detailed experiments with directly reconstructed $3 \mathrm{D}$ grain orientation distribution would be required to quantitatively verify our 
simulation results.

The grain size evolution of Ni/LSM phase in the anode and cathode at different compositions are given in Figs. 6(c) and 6(d). It demonstrates that the grain size evolution rate of the solid phase is sensitive to the composition of the electrode. The Ni/LSM grains in the electrode with more electrode phase reach greater average grain size in the same time interval. Similar dependence on the total volume fraction has been found in the size evolution of YSZ grains as well. It has been found in experiments that the coarsening rate of the electrode phase increases with increased volume fraction of electrode phase in Ni-YSZ anode [60, 61] and LSM-CGO cathode [47], which has been attributed to the blocking effect of the static electrolyte phase. Our simulations show that this composition effect on the coarsening rate of electrode phase still exists even when the electrolyte phase is evolving. This composition effect is a result of the changing in the effective diffusion length. The decreasing in the volume fraction of the electrolyte phase generally reduces the distance between two particles of the electrode phase. This shortens the effective diffusion length and leads to a faster rate of the coarsening of the electrode phase. This composition effect indicates that the coarsening rate of the electrode phase could be controlled by tuning the composition of the electrodes.

\subsection{Triple phase boundary}

The time evolution of TPB density is extracted from the simulated microstructures. The TPB density is calculated by dividing the total length of TPB segments by the volume of the simulation cell. In the cubic mesh grid used in this work, each edge of a cubic unit cell is shared by four nearest neighbor unit cells. An edge is considered as a TPB segment if there are three phases in four of its nearest neighbor unit cells. But if one phase occupies a pair of diagonal nearest neighbor unit cells of an edge, it is not considered as a TPB segment for that this pair of diagonal sites could be connected on a denser mesh grid.

The time evolution of TPB density in SOFC electrodes with 35\% YSZ, 35\% 
LSM and 30\% Pore is given in Fig. 6(e). The TPB evolution in the electrodes with other composition is similar. It shows that the TPB density decreases with time in all electrodes. After 110 hours, the TPB density reduces from $\sim 1.8 \mu \mathrm{m}^{-2}$ to $\sim 0.9 \mu \mathrm{m}^{-2}$ for the anode and $\sim 1.0 \mu \mathrm{m}^{-2}$ for the cathode and the reference electrode. This TPB density range is in good agreement with the reconstructed anode microstructure with particle size around $0.5 \mu \mathrm{m}$, but the simulated degradation rate of TPB density in anode ( 50\% for 110 hours at 1000 ${ }^{\circ} \mathrm{C}$ ) is overestimated compared to experimental observation ( $\sim 30 \%$ for 100 hours at around $\left.1000{ }^{\circ} \mathrm{C}\right)[12,62,63]$. This overestimation in TPB degradation rate is a result of the exaggerated mobility of YSZ phase. Comparable degradation rate of TPB density can be obtained by slowing down the evolution of YSZ phase as discussed in Sec. 5.1.

The degradation behavior of TPB shows clear dependence on the interfacial energetics. In all compositions, the TPB density in the anode at the end of the simulation is found to be lower than that in the cathode and reference electrode by around $10 \%$. And the degradation of TPB density in the cathode and reference electrode are almost the same. Since the GB energies of both electrolyte and electrode phases in the anode are the lowest in three representative electrodes, it shows that the degradation of TPB density can be slowed down by increasing the GB energy of solid phases in SOFC electrodes.

\subsection{Effective conductivity}

The time evolution of the normalized effective conductivity of SOFC electrodes are shown in Fig. 6(f), where $\sigma_{m f}$ is the mean field conductivity, which is used as a normalization factor and is calculated by averaging the bulk conductivity of YSZ and Ni/LSM weighted by their total volume fraction. It shows that the electrodes with more Ni/LSM have greater effective conductivity. With the total Ni/LSM volume fraction increases from $30 \%$ to $40 \%$, the effective conductivity increases from around $0.3 \sigma_{m f}$ to around $0.5 \sigma_{m f}$. It is found that the value of the effective conductivity is more than doubled when Ni/LSM content increases from $30 \%$ to $40 \%$ considering that $\sigma_{m f}$ also increases with increasing 
$\mathrm{Ni} / \mathrm{LSM}$ content.

The effective conductivity decreases with time for all cases tested, which is consistent with previous phase field simulations [25] and experiments [18, 20, 64] on the electronic conductivity of the electrode phase. After 110 hours, the effective conductivity reduces by around $0.03 \sigma_{m f}$ for the anode, $0.10 \sigma_{m f}$ for the cathode and $0.08 \sigma_{m f}$ for the reference electrode. It is clear that the conductivity degradation rate is the lowest in the anode and the highest in the cathode, which demonstrates the effect of the GB energy of the electrode phase on the electrode degradation. Although the effective conductivity is dominated by the electronic conductivity of the electrode phase, we expect that similar loss of ionic conductivity can be found on the YSZ network for that the size evolution of the YSZ phase is similar to the Ni phase. Our results suggest that a lower GB energy of the electrode/electrolyte phase is beneficial to lower the degradation rate of the electronic/ionic conductivity of SOFC electrodes.

\section{Discussion}

\subsection{Effect of mobility}

A major task of this work is to enhance the tunability of the energetics of previous phase field models, and so far the mobility of each phase is simply set to a constant. This simplification has two problems. The first is that the cation diffusivity in YSZ is much smaller than the self-diffusivity of $\mathrm{Ni}$ and the cation diffusivity in LSM [21, 25]. So setting the mobility of YSZ equal to the mobility of Ni/LSM overestimates the particle coarsening of YSZ in SOFC electrodes. The second is that the mobility of one phase should be different in different phases. The blocking effect due to the mobility difference in different phases has been ignored when the mobility is set to a constant. Therefore, we carry out two test simulations on the anode with $35 \%$ YSZ, 35\% Ni and 30\% pores to demonstrate the effect of mobility on the grain size evolution. The time evolution of average $\mathrm{Ni}$ grain radius and TPB density in these two simulations are given in Figs. 7(a) and 7(b), and compared to the simulation with constant mobility. 
The first test simulation assumes that the evolution of the YSZ phase stopped after 5000 time steps to reflect the much slower grain size evolution in YSZ. The lines with diamond marker in Figs. 7(a) and 7(b) show the time evolution of $\mathrm{Ni}$ grain radius and TPB density for this test simulation. Compared to the simulation with constant mobility, it shows that the size evolution of $\mathrm{Ni}$ grains and the degradation of the anode has been slowed down by the static YSZ grains. The final average radius of $\mathrm{Ni}$ reduces from $\sim 0.75 \mu \mathrm{m}$ with constant mobility to $\sim 0.64 \mu \mathrm{m}$ with static YSZ, while the degradation of TPB density reduces from $\sim 50 \%$ with constant mobility to $\sim 30 \%$ with static YSZ after 110 hours at $1000{ }^{\circ} \mathrm{C}$. The resulting degradation rate of TPB density with static YSZ is in good agreement with experiments $[12,62,63]$. This shows that the pinning effect of YSZ grains on the size evolution of Ni grain is important to the microstructure evolution of anode.

The second test simulation assumes that the mobility of YSZ $(\mathrm{Ni})$ in $\mathrm{Ni}$ (YSZ) and pore is one and two orders of magnitude lower than the mobility of YSZ (Ni) in YSZ (Ni) to reflect the blocking effect of other phases. The lines with triangle marker in Fig. 7(a) and 7(b) show the time evolution of $\mathrm{Ni}$ grain radius and TPB density for this test simulation. Similar to the first test simulations, the smaller mobility of YSZ (Ni) in Ni (YSZ) and pore slows down the degradation of the anode. After 110 hours, the final the average radius of $\mathrm{Ni}$ grains is $\sim 0.60 \mu \mathrm{m}$ and the degradation of TPB density is $\sim 35 \%$ in this test simulation compared to $\sim 0.75 \mu \mathrm{m}$ and $\sim 50 \%$ in the simulation with constant mobility.

Despite the difference in the grain size evolution and degradation rate, the microstructure evolution in these two test simulations resembles the same general features, e.g. the size of Ni grains increases faster than that of YSZ and consistent TPB density reducing, as the simulation with constant mobility. This shows that a qualitative description of the microstructure evolution in SOFC electrodes has already been obtained by incorporating the appropriate interfacial energetics. Moreover, these two test simulations demonstrate that the material dependent mobility can be handled by our model once the mobility of 
all materials in all phases are known.
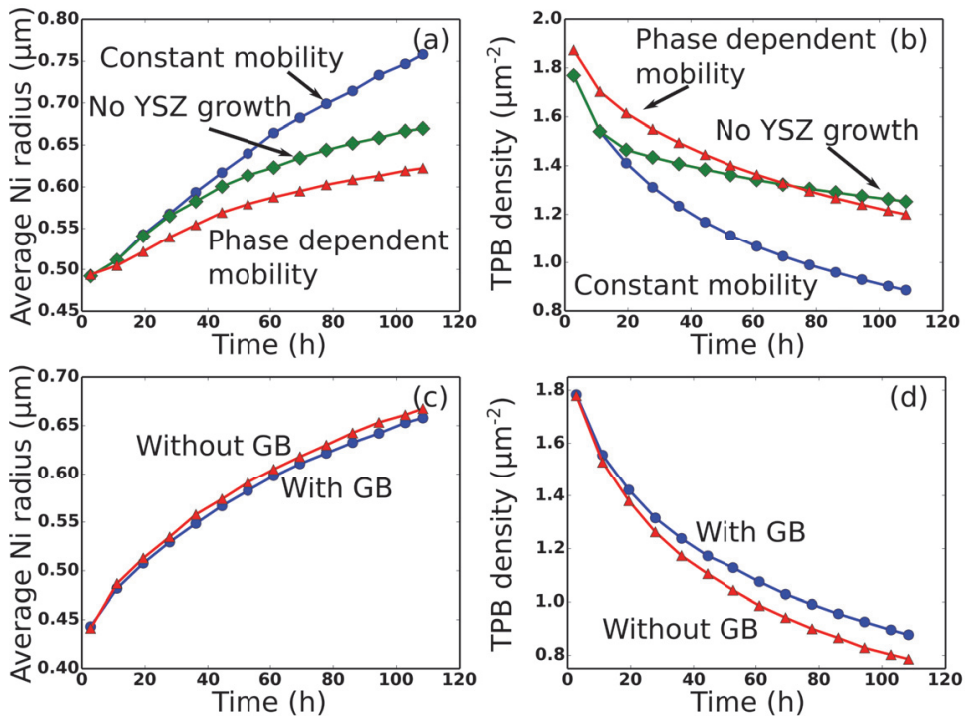

Figure 7: The time evolution of (a) the average Ni grain radius and (b) TPB density in the anode with three different mobility setups. And the time evolution of (c) the average Ni grain radius and (d) TPB density in the anode with and without GB. The composition of the anode is $35 \% \mathrm{YSZ}, 35 \% \mathrm{Ni}$ and $30 \%$ pores.

\subsection{Grain boundary effect}

The GB effect on microstructure evolution has been ignored in most previous phase field models [21-24]. The results in Sec. 4 have already shown that GB energy can affect overall microstructure evolution rate including the TPB density degradation rate. Without considering GB is equivalent to set GB en-

ergy as zero. Therefore, we expect that ignoring GB would overestimate the rate of microstructure evolution in SOFC electrode. In Figs. 7(c) and 7(d), we compare two test simulations on the anode with and without GB at the composition of 35\% YSZ, 35\% Ni and 30\% pores. The simulation without GB has been performed by using only one GOP $\eta$ for each solid phase. Inscribed sphere is used to determine the average grain radius of each phase in both simulations in order to compare them. Fig. 7(c) shows that the size of Ni grains increases faster in the simulation without GB than that with GB. Similar GB effect has been found in the grain size evolution of YSZ and pore phase as well. In addi- 
tion, Fig. 7(d) shows that the TPB density in the anode decreases faster over time in the simulations without GB. It is clear that ignoring GBs leads to an overestimation of the rate of microstructure evolution in phase field simulations. Detailed analyses show that GBs change the local curvature of the surfaces, so ignoring GBs not only overestimates the grain growth rate but also the particle coarsening rate. This overestimation of the rate of microstructure evolution might not have a significant effect in short term, but can have a non-negligible impact on predicting long term properties of the electrode. Therefore, for the purpose of simulating long term degradation of SOFC electrodes, it is essential to incorporate the GB effect even when the elimination of GB is not the major driving force for the microstructure evolution.

\subsection{Effective conductivity vs microstructure evolution}

For the purpose of understanding the evolution of the effective conductivity, we calculate the time evolution of the percolated volume fraction and the geometric tortuosity of the electrode phase. The electrode phase is chosen for that the effective conductivity is dominated by the electronic conductivity provided by its conductive network. The loss of the percolated Ni volume fraction is believed to be the reason of the reduced conductivity in the anode [64], while the geometric tortuosity is considered as an important factor for the transport properties of the porous medium [65]. It is found that the percolated volume fraction of the electrode phase decreases by $1 \% \sim 8 \%$ while the geometric tortuosity of the electrode phase increases by 1\% 7\% after 110 hours. These changes are relatively small compared to the loss of effective conductivity. However, they imply that the connection between the grains of the electrode phase is getting poorer because of the microstructure evolution. The correlation between the effective conductivity, the percolated volume fraction and the geometric tortuosity of the electrode phase are shown in Fig. 8. It shows that the effective conductivity is greater when the percolated volume fraction of the electrode phase is greater and the tortuosity of the electrode phase is smaller. This indicates that the reduced effective conductivity is a result of the loss of connection in the electrode 
phase due to the microstructure evolution.
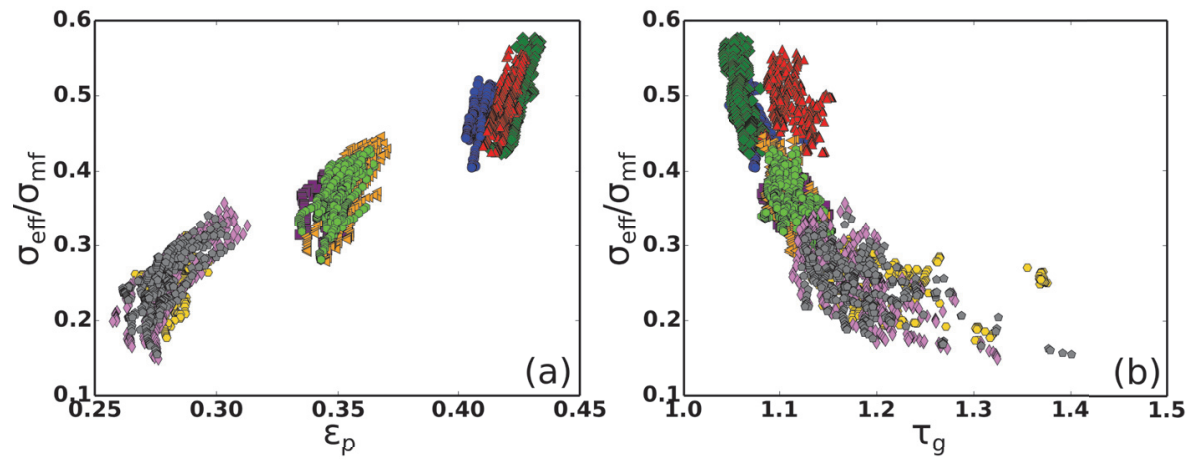

- Anode, 30\%YSZ-40\%LSM-30\%Pore Anode, 35\%YSZ-35\%LSM-30\%Pore O Anode, 40\%YSZ-30\%LSM-30\%Pore

- Cathode, 30\%YSZ-40\%LSM-30\%Pore $\triangleleft$ Cathode, 35\%YSZ-35\%LSM-30\%Pore Cathode, 40\%YSZ-30\%LSM-30\%Pore

$\Delta$ Reference, 30\%YSZ-40\%LSM-30\%Pore Reference, 35\%YSZ-35\%LSM-30\%Pore - Reference, 40\%YSZ-30\%LSM-30\%Pore

Figure 8: The correlation between the effective conductivity and (a) the percolated volume fraction $\left(\varepsilon_{p}\right)$, and (b) the geometric tortuosity $\left(\tau_{g}\right)$ of the electrode phase. (For color version of this figure, the reader is referred to the online version of this article.)

\section{Conclusions}

In this work, we propose an improved multiphase-multigrain phase field model for simulating the microstructure evolution of SOFC electrode. Three sets of parameters for representative YSZ/Ni anode, YSZ/LSM cathode and a reference electrode are developed showing the enhanced tunability of the interfacial energies in the model. The contact angles at triple junctions of these three electrodes have been calibrated conforming to Young's equation. The kinetics of this model is validated by simulating the grain growth and particle coarsening due to Ostwald ripening in 2D. The exponent of the time evolution of grain radius is in good agreement with theory and previous simulations.

Three dimensional microstructure evolution of SOFC electrodes is simulated using this model. The time evolution of some critical geometric properties such as average radius, TPB density and tortuosity are obtained using this model. Though quantitative simulation of the microstructure evolution has not been done due to the lack of mobility data of each phase. We demonstrate that our model is capable of simulating the microstructure evolution with different mo- 
bility for different phases. In the meantime, setting the mobility of all phases as a constant manifests the role of interfacial energies in microstructure evolution. Getting realistic interfacial energy and mobility of the related materials from either experiments or atomistic simulations is essential to link the simulated microstructure evolution to real electrode.

In addition, we show that BCSA algorithm can be generalized to calculate the effective conductivity of SOFC electrodes. The time evolution of effective conductivity is obtained from the simulated microstructure and is found to decrease with time. This is consistent with the loss of percolated volume fraction and the increased tortuosity of the electrode phase which indicates the loss of connection during the microstructure evolution. This technique can be further generalized to simulate other transport properties such as effective diffusivity of SOFC electrodes. The continuation of this work will help in understanding the long-term degradation of SOFC and searching for optimizing materials and initial microstructures for SOFC electrodes.

\section{Acknowledgments}

The authors are grateful to Drs. Kirk Gerdes, Thomas Kalapos, Jason Vielma (NETL) and Paul Salvador (Carnegie Mellon University) for valuable discussions, and Drs. Harry Abernathy, Gregory Hackett (NETL) and Professor Long-Qing Chen (Pennsylvania State University) for critically reading the manuscript. We also thank Dr. William Epting (NETL) for the support of inscribed sphere and tortuosity calculations. This project is supported in part by an appointment to the Internship/Research Participation Program at the National Energy Technology Laboratory, U.S. Department of Energy, administered by the Oak Ridge Institute for Science and Education.

Disclaimer: "This report was prepared as an account of work sponsored by an agency of the United States Government. Neither the United States Government nor any agency thereof, nor any of their employees, makes any warranty, 
express or implied, or assumes any legal liability or responsibility for the accuracy, completeness, or usefulness of any information, apparatus, product, or process disclosed, or represents that its use would not infringe privately owned rights. Reference herein to any specific commercial product, process, or service by trade name, trademark, manufacturer, or otherwise does not necessarily constitute or imply its endorsement, recommendation, or favoring by the United States Government or any agency thereof. The views and opinions of authors expressed herein do not necessarily state or reflect those of the United States Government or any agency thereof." 


\section{Appendix A. Tuning the parameters}

In this appendix, we discuss how to tune the parameters for a specific material system. The target is to tune the relative interfacial energy while keep the width of the interface reasonable for computational efficiency consideration. First, we derive an analytic equation that relates the gradient energy coefficients to the interfacial energy. This equation reveals that, in the original model in Refs. [27] and [28], the energy of IPB is always close to the summation of the surface energy of the two phases forming the IPB. It also reveals that if a GB is formed between two grains with $\eta=1$ and $\eta=-1$ for the same $\eta$, the energy of this GB will be two times of the energy of the GB formed between two grains with $\eta_{1}=1$ and $\eta_{2}=1$. Then, we show that the width of interface can be tuned by adjusting the phenomenological parameters in the local free energy density. A summary of the effect of each parameter on the energy and width of interface is given in Table A.4.

Table A.4: The effect on the energy and width of each interface when a phenomenological parameter is increased. $\uparrow$ and $\downarrow$ means increase and decrease respectively, - means minor effect

\begin{tabular}{ccccccc}
\hline & \multicolumn{2}{c}{ Surface } & \multicolumn{2}{c}{ GB } & \multicolumn{2}{c}{ IPB } \\
& energy & width & energy & width & energy & width \\
\hline$\kappa_{C}^{i i}$ & $\uparrow$ & $\uparrow$ & - & - & $\uparrow$ & $\uparrow$ \\
$\kappa_{C}^{12}$ & - & - & - & - & $\downarrow$ & $\downarrow$ \\
$\kappa_{\eta}^{i j}$ & $\uparrow$ & $\uparrow$ & $\uparrow$ & $\uparrow$ & $\uparrow$ & $\uparrow$ \\
$\rho_{i}$ & $\uparrow$ & $\downarrow$ & - & - & $\uparrow$ & $\downarrow$ \\
$\rho_{12}$ & $\uparrow$ & $\downarrow$ & - & - & - & - \\
$\chi_{i}$ & - & - & $\uparrow$ & $\downarrow$ & - & - \\
$\epsilon_{j k}^{i}$ & - & - & $\uparrow$ & $\downarrow$ & - & - \\
\hline
\end{tabular}


Appendix A.1. Analytic derivation of interfacial energy

From Eq. 1, the energy of a flat interface in the $y z$-plane is

$$
\begin{aligned}
\gamma_{i n t} & =\int_{-\infty}^{+\infty}\left[f\left(C_{i}, \eta_{j}^{i}\right)-f_{b}\right] d x \\
& =\int_{-\infty}^{+\infty}\left[\Delta f_{0}\left(C_{i}, \eta_{j}^{i}\right)+\sum_{i=1}^{2} \sum_{j=i}^{2} \frac{\kappa_{C}^{i j}}{2}\left(\frac{d C_{i}}{d x} \frac{d C_{j}}{d x}\right)\right. \\
& \left.+\sum_{i=1}^{2} \sum_{j=1}^{M_{i}} \frac{\kappa_{\eta}^{i j}}{2}\left(\frac{d \eta_{j}^{i}}{d x}\right)^{2}\right] d x
\end{aligned}
$$

where $f_{b}$ is the free energy of bulk phase and $\Delta f_{0}\left(C_{i}, \eta_{j}^{i}\right)=f_{0}\left(C_{i}, \eta_{j}^{i}\right)-f_{b}$ is the contribution of the bulk free energy to the interfacial energy. At equilibrium, the interfacial energy is minimized. Therefore,

$$
\frac{\delta \gamma_{i n t}}{\delta C_{i}}=\frac{\delta \gamma_{i n t}}{\delta \eta_{j}^{i}}=0
$$

So,

$$
\begin{aligned}
\frac{\partial \Delta f_{0}^{e q}\left(C_{i}, \eta_{j}^{i}\right)}{\partial C_{i}} & =\kappa_{C}^{i i} \frac{d^{2} C_{i}}{d x^{2}}+\frac{\kappa_{C}^{i j}}{2} \frac{d^{2} C_{j}}{d x^{2}}, i \neq j \\
\frac{\partial \Delta f_{0}^{e q}\left(C_{i}, \eta_{j}^{i}\right)}{\partial \eta_{j}^{i}} & =\kappa_{\eta}^{i j} \frac{d^{2} \eta_{j}^{i}}{d x^{2}}
\end{aligned}
$$

Integrating the total derivative of $\Delta f_{0}\left(C_{i}, \eta_{k}^{i}\right)$ using Eqs. A.4 and A.5, we obtain

$$
\Delta f_{0}^{e q}\left(C_{i}, \eta_{j}^{i}\right)=\sum_{i=1}^{2} \sum_{j=i}^{2} \frac{\kappa_{C}^{i j}}{2}\left(\frac{d C_{i}}{d x} \frac{d C_{j}}{d x}\right)+\sum_{i=1}^{2} \sum_{j=1}^{M_{i}} \frac{\kappa_{\eta}^{i j}}{2}\left(\frac{d \eta_{j}^{i}}{d x}\right)^{2}
$$

Substitute Eq. A.6 into Eq. A.2,

$$
\begin{aligned}
\gamma_{i n t} & =2 \int_{-\infty}^{+\infty} \Delta f_{0}^{e q}\left(C_{i}, \eta_{j}^{i}\right) d x \\
& =\int_{-\infty}^{+\infty}\left[\sum_{i=1}^{2} \sum_{j=i}^{2} \kappa_{C}^{i j}\left(\frac{d C_{i}}{d x} \frac{d C_{j}}{d x}\right)+\sum_{i=1}^{2} \sum_{j=1}^{M_{i}} \kappa_{\eta}^{i j}\left(\frac{d \eta_{j}^{i}}{d x}\right)^{2}\right] d x
\end{aligned}
$$

Eqs. A.7 and A.8 reveals the importance of the term $\left(\nabla C_{1} \cdot \nabla C_{2}\right)$ in Eq. 1. 
It is easy to see that on surface and IPB, Eq. A.8 becomes

$$
\begin{aligned}
\gamma_{S} & =\int_{-\infty}^{+\infty}\left[\kappa_{C}^{i i}\left(\frac{d C_{i}}{d x}\right)^{2}+\kappa_{\eta}^{i j}\left(\frac{d \eta_{j}^{i}}{d x}\right)^{2}\right] d x \\
\gamma_{I P B} & =\int_{-\infty}^{+\infty}\left[\kappa_{C}^{11}\left(\frac{d C_{1}}{d x}\right)^{2}+\kappa_{C}^{22}\left(\frac{d C_{2}}{d x}\right)^{2}+\kappa_{C}^{12}\left(\frac{d C_{1}}{d x} \frac{d C_{2}}{d x}\right)\right. \\
& \left.+\kappa_{\eta}^{1 k}\left(\frac{d \eta_{k}^{1}}{d x}\right)^{2}+\kappa_{\eta}^{2 l}\left(\frac{d \eta_{l}^{2}}{d x}\right)^{2}\right] d x
\end{aligned}
$$

In the formula of Refs. [27] and [28], $\kappa_{C}^{12}$ is 0 . It is clear that the energy of IPB is always close to the summation of the surface energy of phase 1 and 2 in this case, indicating that the IPB is likely to be wet by the pore phase. Only when $\kappa_{C}^{12}>0$, the energy of IPB can be similar to the surface energy of phase 1 and phase 2. Thus, the term $\left(\nabla C_{1} \cdot \nabla C_{2}\right)$ is essential for simulating a stable three phase system.

Further, Eqs. A.8 reveals the importance of eliminating the local minima of the bulk free energy density (Eq. 2) at $C=1$ and $\eta=-1$. From Eq. A.8, when a GB is forming between two grains with $\eta_{j}^{i}=1$ and $\eta_{j}^{i}=-1$ of the same $\eta_{j}^{i}$, its energy is

$$
\gamma_{G B}^{1}=\int_{-\infty}^{+\infty}\left[\kappa_{C}^{i i}\left(\frac{d C_{i}}{d x}\right)^{2}+\kappa_{\eta}^{i j}\left(\frac{d \eta_{j}^{i}}{d x}\right)^{2}\right] d x
$$

For a GB forming between two grains with $\eta_{k}^{i}=1$ and $\eta_{l}^{i}=1$, however, its energy is

$$
\gamma_{G B}^{2}=\int_{-\infty}^{+\infty}\left[\kappa_{C}^{i i}\left(\frac{d C_{i}}{d x}\right)^{2}+\kappa_{\eta}^{i k}\left(\frac{d \eta_{k}^{i}}{d x}\right)^{2}+\kappa_{\eta}^{i l}\left(\frac{d \eta_{l}^{i}}{d x}\right)^{2}\right] d x
$$

$C_{i}$ is only slightly deviated from 1 near GB plane, so the first term of Eqs. A.11 and A.12 can be ignored. Considering that $\eta_{j}^{i}$ changes from +1 to -1 while $\eta_{k}^{i}$ and $\eta_{l}^{i}$ only changes from 1 to 0 across the GB plane, it is reasonable to assume that

$$
\int\left(\frac{d \eta_{j}^{i}}{d x}\right)^{2} d x=4 \int\left(\frac{d \eta_{k}^{i}}{d x}\right)^{2} d x=4 \int\left(\frac{d \eta_{l}^{i}}{d x}\right)^{2} d x
$$

when these two types of GB have similar width. Thus, $\gamma_{G B}^{1}$ is approximately two times of $\gamma_{G B}^{2}$ when all $\kappa_{i j}$ are set to the same value. Therefore, it is essential to 
eliminate the local minima at $C=1$ and $\eta=-1$ of the bulk free energy density to make the energy of all interfaces tunable.

Furthermore, Eqs. A.7 and A.8 can be used to estimate the error of the interfacial energy calculations. The results of the numerical test shown in Table A.5 show that the interfacial energy calculated by Eq. A.2 is always bounded by Eqs. A.7 and A.8. It can be shown that the difference between Eqs. A.7 and A.8 is related to the numerical error of Eq. A.2. Though the exact relation depends on the numerical scheme used in evaluating the integration and gradient, this difference provides an estimation of the uncertainty of the calculated interfacial energy.

Table A.5: Interfacial energies calculated from Eqs. A.7 and A.8 as compared to Eq. A.2 for the anode and cathode material system.

\begin{tabular}{lcccccc}
\hline & \multicolumn{3}{c}{ Anode } & \multicolumn{3}{c}{ Cathode } \\
& Eq. A.7 & Eq. A.8 & Eq. A.2 & Eq. A.7 & Eq. A.8 & Eq. A.2 \\
\hline$\gamma_{s}^{Y S Z}$ & 0.181 & 0.193 & 0.187 & 0.172 & 0.183 & 0.177 \\
$\gamma_{G S Z}^{Y S B}$ & 0.106 & 0.106 & 0.106 & 0.104 & 0.106 & 0.105 \\
$\gamma_{s}^{L S M}$ & 0.296 & 0.317 & 0.306 & 0.179 & 0.200 & 0.190 \\
$\gamma_{G B}^{L S M}$ & 0.143 & 0.143 & 0.143 & 0.244 & 0.263 & 0.253 \\
$\gamma_{I P B}$ & 0.285 & 0.292 & 0.289 & 0.188 & 0.189 & 0.189 \\
\hline
\end{tabular}

The range of $\kappa_{C}^{12}$ can be determined in the following way. Supposing the free energy density of a system with 3 phases is

$$
f=f_{0}+\frac{\alpha}{2}\left(\nabla C_{1}\right)^{2}+\frac{\beta}{2}\left(\nabla C_{2}\right)^{2}+\frac{\gamma}{2}\left(\nabla C_{3}\right)^{2}
$$

where $\alpha, \beta$ and $\gamma$ are the gradient energy coefficients whose value should be positive, then, since $C_{1}+C_{2}+C_{3}=1$, we have

$$
f=f_{0}+\frac{\alpha+\gamma}{2}\left(\nabla C_{1}\right)^{2}+\frac{\beta+\gamma}{2}\left(\nabla C_{2}\right)^{2}+\gamma\left(\nabla C_{1} \cdot \nabla C_{2}\right)
$$

Comparing Eq. A.15 to Eq. 1, it is easy to see that

$$
\alpha+\gamma=\kappa_{C}^{11}, \beta+\gamma=\kappa_{C}^{22}, \gamma=\frac{\kappa_{C}^{12}}{2}
$$

Because that $\alpha, \beta$ and $\gamma$ should all be positive, we have

$$
0<\frac{\kappa_{C}^{12}}{2}<\min \left(\kappa_{C}^{11}, \kappa_{C}^{22}\right)
$$




\section{Appendix A.2. Width of interfaces}

The above section discusses how to tune the interfacial energy by adjusting the gradient energy coefficients $\kappa_{C}^{i j}$ and $\kappa_{\eta}^{i j}$. In addition, the width of the interface needs to be tuned as well to keep computational efficiency of the phase field modeling. Thus, it is necessary to keep the width as small as possible, while avoid extremely narrow interface, which is artificially slow to move due to numerical issue [66]. This can be done by adjusting the phenomenological parameters $\rho_{i}, \rho_{12}, \chi_{i}$ and $\epsilon_{j k}^{i}$ in Eq. 2 .

It has been shown in previous phase field simulations that the width of the interface is inversely proportional to the potential depth on the interface [66, 67]. In the bulk free energy functional as shown in Eq. $2, f_{2}\left(C_{i}\right)$ and $f_{3}\left(C_{i}, \eta_{j}^{i}\right)$ describe the interaction between $C_{i}$ and $\eta_{j}^{i}$, which has limited effect on the potential depth on interfaces. Among other terms, the potential depth on GB can be independently tuned by adjusting the coefficient for $f_{4} \epsilon_{j k}^{i}$, while the potential depths on surface and IPB are controlled by $\rho_{1}, \rho_{2}$ and $\rho_{12}$. On the surface of phase 1, the first three terms of Eq. 2 reduce to

$$
f_{S}\left(C_{1}\right)=\left(\rho_{1}+\rho_{12}\right) C_{1}^{2}\left(1-C_{1}\right)^{2}
$$

which is a simple double well with the potential depth of $2\left(\rho_{1}+\rho_{12}\right)$. On the interphase boundary, supposing $C_{1}+C_{2}=1$, the first three terms of Eq. 2 become

$$
f_{I P B}\left(C_{1}\right)=\left(\rho_{1}+\rho_{2}\right) C_{1}^{2}\left(1-C_{1}\right)^{2}
$$

which is a simple double well with the potential depth of $2\left(\rho_{1}+\rho_{2}\right)$. In the formula of Refs. [27] and [28], there is no term $\rho_{12} f_{1}\left(C_{1}+C_{2}\right)$ but have a term $\frac{\lambda}{2} C_{1}^{2} C_{2}^{2}$. Since $C_{1}^{2} C_{2}^{2}$ is equal to zero when $C_{1}=0$ or $C_{2}=0$ but is always greater than 0 when $C_{1} \neq 0$ and $C_{2} \neq 0$, it is easy to see that in the formula of Refs. [27] and [28], the potential depth on IPB is always greater than the summation of the potential depth on the surface of phase 1 and 2, which can lead to an extremely narrow IPB. Thus, the term $f_{1}\left(C_{1}+C_{2}\right)$ is essential to make the width of all interfaces similar. 
Eqs. A.18 and A.19 show that the potential depth on the surfaces and IPB can be tuned by adjusting $\rho_{1}, \rho_{2}$ and $\rho_{12}$. Their ranges can be determined in the following way. It can be proven that the function

$$
\begin{aligned}
f\left(C_{1}, C_{2}\right) & =\rho_{1} C_{1}^{2}\left(1-C_{1}\right)^{2}+\rho_{2} C_{2}^{2}\left(1-C_{2}\right)^{2} \\
& +\rho_{12}\left(C_{1}+C_{2}\right)^{2}\left(1-C_{1}-C_{2}\right)^{2}
\end{aligned}
$$

has three stationary points at $(0.5,0),(0,0.5)$ and $(0.5,0.5)$ which correspond to the surface of phase 1 , surface of phase 2 and interphase boundary, respectively. To make these interfaces stable, it is essential for these points to be a first order saddle point on the $C_{1}-C_{2}$ plane. This leads to the three constrains on the value of $\rho_{1}, \rho_{2}$ and $\rho_{12}$

$$
\begin{array}{r}
2 \rho_{1} \rho_{2}+\left(2 \rho_{2}-\rho_{1}\right) \rho_{12}>0 \\
2 \rho_{1} \rho_{2}+\left(2 \rho_{1}-\rho_{2}\right) \rho_{12}>0 \\
\rho_{1} \rho_{2}-2\left(\rho_{1}+\rho_{2}\right) \rho_{12}<0
\end{array}
$$

\section{References}

[1] D. Ding, X. Li, S. Y. Lai, K. Gerdes, M. Liu, Enhancing SOFC cathode performance by surface modification through infiltration, Energy Environ. Sci. 7 (2014) 552-575. doi:10.1039/C3EE42926A.

URL http://dx.doi.org/10.1039/C3EE42926A

[2] T. S. Lee, J. Chung, Y.-C. Chen, Design and optimization of a combined fuel reforming and solid oxide fuel cell system with anode off-gas recycling, Energy Conversion and Management 52 (10) (2011) 3214-3226. doi:http://dx.doi.org/10.1016/j.enconman.2011.05.009.

URL http://www. sciencedirect. com/science/article/pii/S019689041100166X

[3] S. C. Singhal, Solid oxide fuel cells for power generation, Wiley Interdisciplinary Reviews: Energy and Environment 3 (2) (2014) 179-194. doi:10.1002/wene.96.

URL http://dx.doi.org/10.1002/wene.96 
[4] A. Choudhury, H. Chandra, A. Arora, Application of solid oxide fuel cell technology for power generation-a review, Renewable and Sustainable Energy Reviews 20 (2013) 430-442. doi:http://dx.doi.org/10.1016/j.rser.2012.11.031.

URL http://www. sciencedirect.com/science/article/pii/S1364032112006430

[5] M. Liu, M. E. Lynch, K. Blinn, F. M. Alamgir, Y. Choi, Rational SOFC material design: new advances and tools, Materials Today 14 (11) (2011) 534-546. doi:http://dx.doi.org/10.1016/S1369-7021(11)70279-6.

URL http://www.sciencedirect.com/science/article/pii/S1369702111702796

[6] J. M. Vohs, R. J. Gorte, High-performance SOFC cathodes prepared by infiltration, Advanced Materials 21 (9) (2009) 943-956. doi:10.1002/adma.200802428.

URL http://dx.doi.org/10.1002/adma. 200802428

[7] M. D. Gross, J. M. Vohs, R. J. Gorte, Recent progress in SOFC anodes for direct utilization of hydrocarbons, J. Mater. Chem. 17 (2007) 3071-3077. doi:10.1039/B702633A.

URL http://dx.doi.org/10.1039/B702633A

[8] N. Mahato, A. Banerjee, A. Gupta, S. Omar, K. Balani, Progress in material selection for solid oxide fuel cell technology: A review, Progress in Materials Science 72 (2015) 141-337. doi:http://dx.doi.org/10.1016/j.pmatsci.2015.01.001.

URL http://www.sciencedirect.com/science/article/pii/S0079642515000195

[9] Y. Tang, H. Zhang, L. Cui, C. Ouyang, S. Shi, W. Tang, H. Li, J.S. Lee, L. Chen, First-principles investigation on redox properties of $m$-doped $\mathrm{CeO}_{2}(m=\mathrm{Mn}, \mathrm{Pr}, \mathrm{Sn}, \mathrm{Zr})$, Phys. Rev. B 82 (2010) 125104. doi:10.1103/PhysRevB.82.125104.

URL http://link.aps.org/doi/10.1103/PhysRevB.82.125104

[10] L. Cui, Y. Tang, H. Zhang, L. G. Hector, C. Ouyang, S. Shi, H. Li, L. Chen, First-principles investigation of transition metal atom $\mathrm{m}(\mathrm{m}=\mathrm{Cu}, \mathrm{Ag}, \mathrm{Au})$ 
adsorption on $\mathrm{CeO}_{2}(110)$, Phys. Chem. Chem. Phys. 14 (2012) 1923-1933. doi:10.1039/C2CP22720G.

URL http://dx.doi.org/10.1039/C2CP22720G

[11] P. Manning, J. Sirman, J. Kilner, Oxygen self-diffusion and surface exchange studies of oxide electrolytes having the fluorite structure, Solid State Ionics 93 (1-2) (1996) 125-132. doi:http://dx.doi.org/10.1016/S01672738(96)00514-0.

URL http://www.sciencedirect.com/science/article/pii/S0167273896005140

[12] D. Kennouche, Y. K. Chen-Wiegart, C. Riscoe, J. Wang, S. A. Barnett, Combined electrochemical and X-ray tomography study of the high temperature evolution of Nickel-Yttria Stabilized Zirconia solid oxide fuel cell anodes, Journal of Power Sources 307 (2016) 604-612. doi:http://dx.doi.org/10.1016/j.jpowsour.2015.12.126.

URL http: //www.sciencedirect.com/science/article/pii/S0378775315307382

[13] L. Holzer, B. Iwanschitz, T. Hocker, B. Mnch, M. Prestat, D. Wiedenmann, U. Vogt, P. Holtappels, J. Sfeir, A. Mai, T. Graule, Microstructure degradation of cermet anodes for solid oxide fuel cells: Quantification of nickel grain growth in dry and in humid atmospheres, Journal of Power Sources 196 (3) (2011) 1279 - 1294. doi:http://dx.doi.org/10.1016/j.jpowsour.2010.08.017. URL http://www.sciencedirect.com/science/article/pii/S0378775310013352

[14] A. Hagen, R. Barfod, P. V. Hendriksen, Y.-L. Liu, S. Ramousse, Degradation of anode supported SOFCs as a function of temperature and current load, Journal of The Electrochemical Society 153 (6) (2006) A1165-A1171. doi:10.1149/1.2193400.

URL http://jes.ecsdl.org/content/153/6/A1165.abstract

[15] L. Helmick, Microstructural characterization of solid oxide fuel cell cathode materials, Ph.D. thesis, Carnegie Mellon University (2010).

URL http://gradworks.umi .com/34/56/3456014.html 
[16] Y. Liu, K. Thydén, M. Chen, A. Hagen, Microstructure degradation of LSM-YSZ cathode in SOFCs operated at various conditions, Solid State Ionics 206 (2012) 97-103. doi:http://dx.doi.org/10.1016/j.ssi.2011.10.020. URL http://www.sciencedirect.com/science/article/pii/S0167273811005273

[17] D. Simwonis, F. Tietz, D. Stöver, Nickel coarsening in annealed Ni/8YSZ anode substrates for solid oxide fuel cells, Solid State Ionics 132 (3-4) (2000) 241-251. doi:http://dx.doi.org/10.1016/S0167-2738(00)00650-0.

URL http://www.sciencedirect.com/science/article/pii/S0167273800006500

[18] M. Pihlatie, A. Kaiser, M. Mogensen, Electrical conductivity of Ni-YSZ composites: Variants and redox cycling, Solid State Ionics 222-223 (2012) 38-46. doi:http://dx.doi.org/10.1016/j.ssi.2012.06.021.

URL http://www.sciencedirect.com/science/article/pii/S0167273812003980

[19] P. Tanasini, M. Cannarozzo, P. Costamagna, A. Faes, J. Van Herle, A. Hessler-Wyser, C. Comninellis, Experimental and theoretical investigation of degradation mechanisms by particle coarsening in SOFC electrodes, Fuel Cells 9 (5) (2009) 740-752. doi:10.1002/fuce.200800192.

URL http://dx.doi.org/10.1002/fuce. 200800192

[20] M. Shah, P. W. Voorhees, S. A. Barnett, Time-dependent performance changes in LSCF-infiltrated SOFC cathodes: The role of nano-particle coarsening, Solid State Ionics 187 (1) (2011) 64-67. doi:http://dx.doi.org/10.1016/j.ssi.2011.02.003.

URL http://www.sciencedirect.com/science/article/pii/S0167273811000658

[21] R. Davis, F. Abdeljawad, J. Lillibridge, M. Haataja, Phase wettability and microstructural evolution in solid oxide fuel cell anode materials, Acta Materialia 78 (2014) 271-281. doi:http://dx.doi.org/10.1016/j.actamat.2014.06.037.

URL http://www.sciencedirect.com/science/article/pii/S1359645414004571

[22] H.-Y. Chen, H.-C. Yu, J. S. Cronin, J. R. Wilson, S. A. Barnett, K. Thorn- 
ton, Ni coarsening in the three-phase solid oxide fuel cell anode-a phasefield simulation studyarXiv:1201.1567.

[23] H.-Y. Chen, H.-C. Yu, J. S. Cronin, J. R. Wilson, S. A. Barnett, K. Thornton, Simulation of coarsening in three-phase solid oxide fuel cell anodes, Journal of Power Sources 196 (3) (2011) 1333-1337. doi:http://dx.doi.org/10.1016/j.jpowsour.2010.08.010.

URL http://www. sciencedirect.com/science/article/pii/S0378775310013285

[24] J. H. Kim, W. K. Liu, C. Lee, Multi-scale solid oxide fuel cell materials modeling, Computational Mechanics 44 (5) (2009) 683-703. doi:10.1007/s00466-009-0402-7.

URL http://dx.doi.org/10.1007/s00466-009-0402-7

[25] F. Abdeljawad, B. Vlker, R. Davis, R. M. McMeeking, M. Haataja, Connecting microstructural coarsening processes to electrochemical performance in solid oxide fuel cells: An integrated modeling approach, Journal of Power Sources 250 (2014) 319-331. doi:http://dx.doi.org/10.1016/j.jpowsour.2013.10.121.

URL http://www.sciencedirect.com/science/article/pii/S0378775313017874

[26] G. J. Nelson, K. N. Grew, J. R. I. Jr., J. J. Lombardo, W. M. Harris, A. Faes, A. Hessler-Wyser, J. V. herle, S. Wang, Y. S. Chu, A. V. Virkar, W. K. Chiu, Three-dimensional microstructural changes in the Ni-YSZ solid oxide fuel cell anode during operation, Acta Materialia 60 (8) (2012) 3491-3500. doi:http://dx.doi.org/10.1016/j.actamat.2012.02.041.

URL http://www.sciencedirect.com/science/article/pii/S1359645412001498

[27] Q. Li, L. Liang, K. Gerdes, L.-Q. Chen, Phase-field modeling of three-phase electrode microstructures in solid oxide fuel cells, Applied Physics Letters 101 (3) (2012) 033909. doi:http://dx.doi.org/10.1063/1.4738230.

URL http://scitation.aip.org/content/aip/journal/apl/101/3/10.1063/1.4738230

[28] L. Liang, Q. Li, J. Hu, S. Lee, K. Gerdes, L.-Q. Chen, Phase field modeling of microstructure evolution of electrocatalyst-infiltrated solid 
oxide fuel cell cathodes, Journal of Applied Physics 117 (6) (2015) 065105.

doi:http://dx.doi.org/10.1063/1.4908281.

URL http://scitation.aip.org/content/aip/journal/jap/117/6/10.1063/1.4908281

[29] Z. Jiao, N. Shikazono, Simulation of the reduction process of solid oxide fuel cell composite anode based on phase field method, Journal of Power Sources 305 (2016) 10-16. doi:http://dx.doi.org/10.1016/j.jpowsour.2015.11.061.

URL http://www.sciencedirect.com/science/article/pii/S0378775315305619

[30] Z. Jiao, T. Shimura, N. Shikazono, Numerical assessment of SOFC anode polarization with microstructure evolution, ECS Transactions 68 (1) (2015) 1281-1289. arXiv:http://ecst.ecsdl.org/content/68/1/1281.full.pdf+html, doi:10.1149/06801.1281ecst.

URL http://ecst.ecsdl.org/content/68/1/1281. abstract

[31] T.-L. Cheng, Y.-H. Wen, Iterative spectral method for solving electrostatic or magnetostatic problems in complex and evolving heterostructures, Phys. Rev. E 91 (2015) 053307. doi:10.1103/PhysRevE.91.053307.

URL http://link.aps.org/doi/10.1103/PhysRevE.91.053307

[32] N. Moelans, B. Blanpain, P. Wollants, Quantitative phase-field approach for simulating grain growth in anisotropic systems with arbitrary inclination and misorientation dependence, Phys. Rev. Lett. 101 (2008) 025502. doi:10.1103/PhysRevLett.101.025502.

URL http://link.aps.org/doi/10.1103/PhysRevLett.101.025502

[33] J. A. Warren, T. Pusztai, L. Környei, L. Gránásy, Phase field approach to heterogeneous crystal nucleation in alloys, Phys. Rev. B 79 (2009) 014204. doi:10.1103/PhysRevB.79.014204.

URL http://link.aps.org/doi/10.1103/PhysRevB.79.014204

[34] V. I. Levitas, A. M. Roy, Multiphase phase field theory for temperature-induced phase transformations: Formulation and application to interfacial phases, Acta Materialia 105 (2016) 244-257. 
doi:http://dx.doi.org/10.1016/j.actamat.2015.12.013.

URL http://www.sciencedirect.com/science/article/pii/S135964541530118X

[35] D. Kennouche, Y. K. Chen-Wiegart, K. J. Yakal-Kremski, J. Wang, J. W. Gibbs, P. W. Voorhees, S. A. Barnett, Observing the microstructural evolution of Ni-Yttria-stabilized zirconia solid oxide fuel cell anodes, Acta Materialia 103 (2016) 204-210. doi:http://dx.doi.org/10.1016/j.actamat.2015.09.055.

URL http://www.sciencedirect.com/science/article/pii/S1359645415007442

[36] S. Vedantam, B. S. V. Patnaik, Efficient numerical algorithm for multiphase field simulations, Phys. Rev. E 73 (2006) 016703. doi:10.1103/PhysRevE.73.016703.

URL http://link.aps.org/doi/10.1103/PhysRevE.73.016703

[37] S. G. Kim, D. I. Kim, W. T. Kim, Y. B. Park, Computer simulations of two-dimensional and three-dimensional ideal grain growth, Phys. Rev. E 74 (2006) 061605. doi:10.1103/PhysRevE.74.061605.

URL http://link.aps.org/doi/10.1103/PhysRevE.74.061605

[38] M. A. Groeber, M. A. Jackson, DREAM.3D: A digital representation environment for the analysis of microstructure in 3D, Integrating Materials and Manufacturing Innovation 3 (1) (2014) 1-17. doi:10.1186/2193-9772-3-5.

URL http://dx.doi.org/10.1186/2193-9772-3-5

[39] A. A. Wheeler, W. J. Boettinger, G. B. McFadden, Phase-field model for isothermal phase transitions in binary alloys, Phys. Rev. A 45 (1992) 74247439. doi:10.1103/PhysRevA.45.7424.

URL http://link.aps.org/doi/10.1103/PhysRevA .45.7424

[40] A. Tsoga, P. Nikolopoulos, Surface and grain-boundary energies in yttriastabilized zirconia (YSZ-8 mol\%), Journal of Materials Science 31 (20) (1996) 5409-5413. doi:10.1007/BF01159310.

URL http://dx.doi.org/10.1007/BF01159310 
[41] H. Meltzman, D. Chatain, D. Avizemer, T. M. Besmann, W. D. Kaplan, The equilibrium crystal shape of nickel, Acta Materialia 59 (9) (2011) 3473-3483. doi:http://dx.doi.org/10.1016/j.actamat.2011.02.021.

URL http://www.sciencedirect.com/science/article/pii/S1359645411001157

[42] D. Prokoshkina, V. Esin, G. Wilde, S. Divinski, Grain boundary width, energy and self-diffusion in nickel: Effect of material purity, Acta Materialia $61 \quad$ (14) (2013) 5188-5197. doi:http://dx.doi.org/10.1016/j.actamat.2013.05.010.

URL http://www.sciencedirect.com/science/article/pii/S1359645413003789

[43] H. Nahor, H. Meltzman, W. D. Kaplan, Ni-YSZ(111) solid-solid interfacial energy, Journal of Materials Science 49 (11) (2013) 3943-3950. doi:10.1007/s10853-013-7960-y.

URL http://dx.doi.org/10.1007/s10853-013-7960-y

[44] P. Shewmon, Diffusion in Solids, 2nd Edition, Wiley-TMS, 1991.

[45] M. Palcut, R. Knibbe, K. Wiik, T. Grande, Cation inter-diffusion between $\mathrm{LaMnO}_{3}$ and $\mathrm{LaCoO}_{3}$ materials, Solid State Ionics 202 (1) (2011) 6 - 13. doi:http://dx.doi.org/10.1016/j.ssi.2011.09.003.

URL http://www.sciencedirect.com/science/article/pii/S0167273811004474

[46] M. Kilo, M. Taylor, C. Argirusis, G. Borchardt, R. Jackson, O. Schulz, M. Martin, M. Weller, Modeling of cation diffusion in oxygen ion conductors using molecular dynamics, Solid State Ionics 175 (14) (2004) 823 - 827, fourteenth International Conference on Solid State Ionics. doi:http://dx.doi.org/10.1016/j.ssi.2004.09.059.

URL http://www.sciencedirect.com/science/article/pii/S0167273804006575

[47] D. W. Ni, K. B. Andersen, V. Esposito, Sintering and grain growth kinetics in $\mathrm{La}_{0.85} \mathrm{Sr}_{0.15} \mathrm{MnO}_{3}-\mathrm{Ce}_{0.9} \mathrm{Gd}_{0.1} \mathrm{O}_{1.95}$ (LSM-CGO) porous composite, Journal of the European Ceramic Society 34 (15) (2014) 3769 - 3778. doi:http://dx.doi.org/10.1016/j.jeurceramsoc.2014.04.044.

URL http://www.sciencedirect.com/science/article/pii/S0955221914002477 
[48] V. Randle, D. Horton, Grain growth phenomena in nickel, Scripta Metallurgica et Materialia 31 (7) (1994) 891 - 895. doi:http://dx.doi.org/10.1016/0956-716X(94)90498-7.

URL http://www.sciencedirect.com/science/article/pii/0956716X94904987

[49] G. Gottstein, L. Shvindlerman, Grain Boundary Migration in Metals: Thermodynamics, Kinetics, Applications, Materials Science \& Technology, Taylor \& Francis, 1999.

URL https://books.google.com/books?id=pKaFe6njzdUC

[50] S. P. S. Badwal, Electrical conductivity of single crystal and polycrystalline yttria-stabilized zirconia, Journal of Materials Science 19 (6) (1984) 17671776. doi:10.1007/BF00550246.

URL http://dx.doi.org/10.1007/BF00550246

[51] H. L. Tuller, Ionic conduction in nanocrystalline materials, Solid State Ionics 131 (12) (2000) 143 - 157. doi:http://dx.doi.org/10.1016/S01672738(00)00629-9.

URL http://www.sciencedirect.com/science/article/pii/S0167273800006299

[52] K. Rhazaoui, Q. Cai, C. Adjiman, N. Brandon, Towards the 3D modeling of the effective conductivity of solid oxide fuel cell electrodes - II. computational parameters, Chemical Engineering Science 116 (2014) 781-792. doi:http://dx.doi.org/10.1016/j.ces.2014.05.045.

URL http://www.sciencedirect. com/science/article/pii/S0009250914002711

[53] B. McCarthy, L. Pederson, Y. Chou, X.-D. Zhou, W. Surdoval, L. Wilson, Low-temperature sintering of lanthanum strontium manganite-based contact pastes for SOFCs, Journal of Power Sources 180 (1) (2008) 294-300. doi:http://dx.doi.org/10.1016/j.jpowsour.2008.01.097.

URL http://www.sciencedirect.com/science/article/pii/S0378775308002097

[54] L.-Q. Chen, W. Yang, Computer simulation of the domain dynamics of a quenched system with a large number of nonconserved order parameters: The grain-growth kinetics, Phys. Rev. B 50 (1994) 15752-15756. 
doi:10.1103/PhysRevB.50.15752.

URL http://link.aps.org/doi/10.1103/PhysRevB.50.15752

[55] D. Fan, S. Chen, L.-Q. Chen, P. Voorhees, Phase-field simulation of 2-D Ostwald ripening in the high volume fraction regime, Acta Materialia 50 (8) (2002) 1895-1907. doi:http://dx.doi.org/10.1016/S1359-6454(01)00393-7. URL http://www.sciencedirect.com/science/article/pii/S1359645401003937

[56] D. Fan, L.-Q. Chen, Diffusion-controlled grain growth in two-phase solids, Acta Materialia 45 (8) (1997) 3297-3310. doi:http://dx.doi.org/10.1016/S1359-6454(97)00022-0.

URL http://www.sciencedirect. com/science/article/pii/S1359645497000220

[57] K. Rhazaoui, Q. Cai, M. Kishimoto, F. Tariq, M. Somalu, C. Adjiman, N. Brandon, Towards the 3D modelling of the effective conductivity of solid oxide fuel cell electrodes-validation against experimental measurements and prediction of electrochemical performance, Electrochimica Acta 168 (2015) 139-147. doi:http://dx.doi.org/10.1016/j.electacta.2015.04.005.

URL http://www.sciencedirect.com/science/article/pii/S001346861500883X

[58] K. Zheng, M. Ni, Reconstruction of solid oxide fuel cell electrode microstructure and analysis of its effective conductivity, Science Bulletin 61 (1) (2016) 78-85. doi:10.1007/s11434-015-0946-1.

URL http://dx.doi.org/10.1007/s11434-015-0946-1

[59] W. K. Epting, J. Gelb, S. Litster, Resolving the three-dimensional microstructure of polymer electrolyte fuel cell electrodes using nanometerscale X-ray computed tomography, Advanced Functional Materials 22 (3) (2012) 555-560. doi:10.1002/adfm.201101525.

URL http://dx.doi.org/10.1002/adfm.201101525

[60] Y.-c. K. Chen-Wiegart, D. Kennouche, J. Scott Cronin, S. A. Barnett, J. Wang, Effect of $\mathrm{Ni}$ content on the morphological evolution of $\mathrm{Ni}$ YSZ solid oxide fuel cell electrodes, Applied Physics Letters 108 (8). 
doi:http://dx.doi.org/10.1063/1.4942459.

URL http://scitation.aip.org/content/aip/journal/apl/108/8/10.1063/1.4942459

[61] S. P. Jiang, Sintering behavior of $\mathrm{Ni} / \mathrm{Y}_{2} \mathrm{O}_{3}-\mathrm{ZrO}_{2}$ cermet electrodes of solid oxide fuel cells, Journal of Materials Science 38 (18) (2003) 3775-3782. doi:10.1023/A:1025936317472.

URL http://dx.doi.org/10.1023/A:1025936317472

[62] J. R. Wilson, J. S. Cronin, S. A. Barnett, Linking the microstructure, performance and durability of Ni-yttria-stabilized zirconia solid oxide fuel cell anodes using three-dimensional focused ion beamscanning electron microscopy imaging, Scripta Materialia 65 (2) (2011) 67 - 72, viewpoint set no. 48: Solid Oxide Fuel Cells. doi:http://dx.doi.org/10.1016/j.scriptamat.2010.09.025.

URL http://www.sciencedirect.com/science/article/pii/S1359646210006433

[63] J. S. Cronin, J. R. Wilson, S. A. Barnett, Impact of pore microstructure evolution on polarization resistance of Ni-Yttria-stabilized zirconia fuel cell anodes, Journal of Power Sources 196 (5) (2011) 2640 - 2643. doi:http://dx.doi.org/10.1016/j.jpowsour.2010.10.084.

URL http://www.sciencedirect.com/science/article/pii/S0378775310018847

[64] M. Pihlatie, A. Kaiser, M. Mogensen, M. Chen, Electrical conductivity of Ni-YSZ composites: Degradation due to $\mathrm{Ni}$ particle growth, Solid State Ionics 189 (1) (2011) 82-90. doi:http://dx.doi.org/10.1016/j.ssi.2011.02.001.

URL http://www.sciencedirect.com/science/article/pii/S0167273811000634

[65] L. Holzer, D. Wiedenmann, B. Münch, L. Keller, M. Prestat, P. Gasser, I. Robertson, B. Grobéty, The influence of constrictivity on the effective transport properties of porous layers in electrolysis and fuel cells, Journal of Materials Science 48 (7) (2013) 2934-2952. doi:10.1007/s10853-012-6968-z. URL http://dx.doi.org/10.1007/s10853-012-6968-z 
[66] D. Fan, L.-Q. Chen, S. Chen, Effect of grain boundary width on grain growth in a diffuse-interface field model, Materials Science and Engineering: A 238 (1) (1997) 78-84. doi:http://dx.doi.org/10.1016/S09215093(97)00437-1.

URL http://www. sciencedirect. com/science/article/pii/S0921509397004371

[67] W. J. Boettinger, J. A. Warren, C. Beckermann, A. Karma, Phase-field simulation of solidification, Annual Review of Materials Research 32 (1) (2002) 163-194. doi:10.1146/annurev.matsci.32.101901.155803.

URL http://dx.doi.org/10.1146/annurev.matsci.32.101901.155803 